\title{
Topological membranes, current algebras and H-flux-R-flux duality based on Courant algebroids
}

\author{
Taiki Bessho, ${ }^{a}$ Marc A. Heller, ${ }^{a}$ Noriaki Ikeda ${ }^{b}$ and Satoshi Watamura ${ }^{a}$ \\ ${ }^{a}$ Particle Theory and Cosmology Group, Department of Physics, \\ Graduate School of Science, Tohoku University, \\ Aoba-ku, Sendai 980-8578, Japan \\ ${ }^{b}$ Department of Mathematical Sciences, Ritsumeikan University, \\ Kusatsu, Shiga 525-8577, Japan \\ E-mail: tbessho@tuhep.phys.tohoku.ac.jp, \\ heller@tuhep.phys.tohoku.ac.jp, nikeda@se.ritsumei.ac.jp, \\ watamura@tuhep.phys.tohoku.ac.jp
}

ABSTRACT: We construct a topological sigma model and a current algebra based on a Courant algebroid structure on a Poisson manifold. In order to construct models, we reformulate the Poisson Courant algebroid by supergeometric construction on a QP-manifold. A new duality of Courant algebroids which transforms $H$-flux and $R$-flux is proposed, where the transformation is interpreted as a canonical transformation of a graded symplectic manifold.

Keywords: Differential and Algebraic Geometry, p-branes, Topological Field Theories, Topological Strings

ArXiv ePrint: 1511.03425 


\section{Contents}

1 Introduction 2

2 Courant algebroids and supergeometry 4

2.1 Courant algebroids 4

2.2 Supergeometric construction $\quad 5$

2.3 QP-manifolds of degree $2 \quad 6$

3 Supergeometric description of Poisson Courant algebroids $\quad 8$

4 Duality between $H$-flux and $R$-flux $\quad 10$

$\begin{array}{lll}\text { 4.1 Flux duality transformations as canonical transformations } & 10\end{array}$

4.2 Two cohomologies on $T^{*}[2] T^{*}[1] M \quad 11$

$\begin{array}{lll}4.3 & \text { Duality of cohomologies } & 12\end{array}$

5 Poisson Courant algebroids from double field theory 13

5.1 Supergeometric formulation, Poisson structure and double field theory 13

$\begin{array}{lll}5.2 & \text { Poisson Courant algebroid } R \text {-flux in double field theory } & 15\end{array}$

6 Topological sigma models $\quad 16$

$\begin{array}{lll}6.1 & \text { AKSZ sigma models } & 16\end{array}$

$\begin{array}{lll}\text { 6.2 AKSZ sigma models with boundary } & 17\end{array}$

$\begin{array}{ll}\text { 6.3 Contravariant Courant sigma models } & 19\end{array}$

6.4 Duality of Courant sigma models 21

$\begin{array}{lll}7 & \text { Current algebras } & 23\end{array}$

$\begin{array}{lll}\text { 7.1 Poisson brackets with fluxes from target QP-structures } & 23\end{array}$

$\begin{array}{ll}7.2 & \text { Current algebras from target QP-structures } \\ 7.36\end{array}$

$\begin{array}{lll}7.3 & \text { Poisson bracket twisted by } R \text {-flux } & 27\end{array}$

$\begin{array}{lll}7.4 & \text { Contravariant current algebras with } R \text {-flux } & 29\end{array}$

8 Conclusions and discussion $\quad 29$

$\begin{array}{ll}\text { A Formulas in graded differential calculus } & 31\end{array}$

A.1 Basic definitions 31

$\begin{array}{lll}\text { A.2 Cartan formulas } & 32\end{array}$

A.3 Differential forms 32

$\begin{array}{lll}\text { A.3.1 Exterior derivatives } & 33\end{array}$

A.4 Graded symplectic form and Poisson bracket 33 


\section{Introduction}

There exist various dualities in string theory. Among them, T-duality is directly connected with the geometry of the target space and thus has to be a characteristic property of stringy geometry.

One of the formulations to analyze T-duality is the approach of doubled geometry, which has manifest $O(d, d)$ invariance, and there, the existence of so-called nongeometric fluxes has been proposed [1]. On the other hand, the fluxes $H, F, Q$ and $R$ and their transformations have also been conjectured from T-duality analysis in supergravity compactification scenario $[2,3]$. It has been proposed that T-duality converts $H$-, $F$-, $Q$ - and $R$-fluxes into each other. Recently, there are further developments related to Tduality. Double field theory [4] is a manifestly $O(d, d)$ covariant field theory which allows also for T-duality along non-isometry directions. Examples for other developments are the branes as sources for $Q$ - and $R$-fluxes [5,6] and the $\beta$-supergravity [7]. The topological T-duality $[8,9]$ is also proposed to analyze T-duality with flux. However, the background geometric structures for nongeometric fluxes are not well understood.

A background geometry in string theory with NS $H$-flux [10] is known to be a Courant algebroid [11, 12], and the standard Courant algebroid of the generalized tangent bundle $T M \oplus T^{*} M$ is of particular interest in the framework of generalized geometry [13, 14]. The T-duality on the $H$-flux is well understood as an automorphism on the standard Courant algebroid if $\iota_{X} \iota_{Y} H=0$ [15].

However, we cannot simultaneously introduce all degrees of freedom of $H_{-}, F_{-}, Q_{-}, R-$ fluxes as deformation of the Courant algebroid. The only independent deformation in the exact Courant algebroid is a 3 -form ( $H$-flux) degree of freedom $[16,17]$.

Recently, the Courant algebroid on a Poisson manifold, i.e. the Poisson Courant algebroid, has been introduced in [18] as a geometric object for a background with $R$-flux. It is shown that the nontrivial flux $R$ of a 3 -vector can be introduced consistently on a Poisson manifold as a deformation of the Courant algebroid. It is the 'contravariant object' [19] with respect to the standard Courant algebroid, which is the exchange of $T^{*} M$ with $T M$ and $H$-flux with $R$-flux. The T-duality on the $R$-flux has also been analyzed and it has been shown that the duality of $R$-flux with $Q$-flux is also understood as an automorphism on the Poisson Courant algebroid [20].

In this paper, we analyze the geometric structure of the Poisson Courant algebroid and a duality between $H$-flux and $R$-flux, which we call flux duality, in detail. We also construct the corresponding worldvolume theories, a topological sigma model and a current algebra with the structure of this Poisson Courant algebroid. 
We first discuss the mathematical features and some structural correspondences between the standard Courant algebroid with $H$-flux and the Poisson Courant algebroid with $R$-flux. By analyzing both coboundary operators, we generalize the duality between the de Rham cohomology and the Poisson cohomology as the background algebraic structure. Moreover, we have a new interpretation of this duality as a canonical transformation on a graded symplectic manifold, ${ }^{1}$ and we formulate a flux duality, a duality between the $H$-flux and $R$-flux.

Then, we discuss field theoretic realizations of the Poisson Courant algebroid as a symmetry; we construct a topological sigma model and a current algebra. To this end, first we reformulate the Courant algebroid in terms of supergeometry. The construction of the Courant algebroid by using supergeometry and the derived brackets are introduced in [23]. This formulation uses a so-called QP-manifold, a differential graded symplectic manifold [24, 25]. The advantage of the use of supergeometry is that the topological sigma model and the current algebra are constructed straightforwardly from the supergeometric data. The general theories are known as the AKSZ construction of topological sigma models [26] and the supergeometric BFV formulation of current algebras [27].

It is known that the AKSZ sigma model in three dimensions generally has the structure of a Courant algebroid [28-30]. Physically, this is a theory of a topological membrane. Following general arguments, we construct a topological sigma model from the Poisson Courant algebroid in three dimensions. When the three-dimensional world volume has a boundary, i.e. when we consider the open membrane, we obtain a two-dimensional boundary sigma model à la WZW. This is the Poisson sigma model with $R$-flux on the Dirac structure of a Poisson Courant algebroid. From the point of view of the sigma model, T-duality is changing the boundary conditions of the topological membranes. There is an approach with a similar concept proposed in [31]. The difference is that our formalism is based on the Poisson Courant algebroid.

We also construct a current algebra of the Poisson Courant algebroid on loop space, coming from the canonical formulation of the theories on $(1+1)$-dimensional spacetime $S^{1} \times \boldsymbol{R}$. In the H-flux case, this is the Alekseev-Strobl current algebra [32], which has the structure of the standard Courant algebroid with H-flux as underlying symmetry. This type of current algebra can also be reformulated by using the supergeometric construction [27, 33]. Following these general formulations, we construct a corresponding current algebra with R-flux.

This paper is organized as follows. In section 2, we review the supergeometric construction of Courant algebroids and, in section 3, we apply it to the Poisson Courant algebroid. In section 4 , we discuss the mathematical structure of the duality of the standard Courant algebroid and the Poisson Courant algebroid. In section 5, we discuss the meaning of $R$-flux of the Poisson Courant algebroid from the perspective of double field theory. In section 6, we review the AKSZ sigma model. Then, we construct a topological sigma model of the Poisson Courant algebroid and analyze its boundary theories. In section 7, we con-

\footnotetext{
${ }^{1}$ T-duality has been formulated as a canonical transformation on the string phase space in [21, 22]. Canonical transformations in this paper are defined on a graded target manifold.
} 
struct the current algebra of the Poisson Courant algebroid. Finally, section 8 is devoted to conclusion and discussion. In the appendix, our notation of supergeometry in this paper is summarized.

\section{Courant algebroids and supergeometry}

In this section, we briefly review supergeometry, its definition and related terms which are necessary to construct the topological sigma models from the standard Courant algebroids using the AKSZ construction in section 5. Here, we review definitions of Courant algebroids in the first subsection. Courant algebroids provide the background geometry of T-duality. The second subsection then reviews a differential graded symplectic manifold, which is called a QP-manifold. In the AKSZ formulation, a QP-manifold is used to construct a topological sigma model. Finally, in the third subsection, the supergeometric construction of the Courant algebroids from QP-manifolds of degree 2 is explained. The formulation is based on the fundamental theorem that general Courant algebroids are equivalent to QPmanifolds of degree 2. This short review of the techniques involved provides the foundation to flow into the definition of Poisson Courant algebroids and their realization through supergeometric construction.

\subsection{Courant algebroids}

Let us start with recalling the definition of the Courant algebroid.

Definition 2.1. [12, 34] The Courant algebroid is a vector bundle $E$ over $M$ with three operations, a pseudo-Euclidean metric $\langle-,-\rangle$ on the fiber, a bundle map $\rho: E \longrightarrow T M$ (called the anchor map), and a binary bracket $[-,-]_{D}$ (the Dorfman bracket) on the space of sections $\Gamma(E)$, which satisfy the following conditions:

$$
\begin{aligned}
& \text { 1) }\left[e_{1},\left[e_{2}, e_{3}\right]_{D}\right]_{D}=\left[\left[e_{1}, e_{2}\right]_{D}, e_{3}\right]_{D}+\left[e_{2},\left[e_{1}, e_{3}\right]_{D}\right]_{D}, \\
& \text { 2) } \rho\left(e_{1}\right)\left\langle e_{2}, e_{3}\right\rangle=\left\langle\left[e_{1}, e_{2}\right]_{D}, e_{3}\right\rangle+\left\langle e_{2},\left[e_{1}, e_{3}\right]_{D}\right\rangle \\
& \text { 3) } \rho\left(e_{1}\right)\left\langle e_{2}, e_{3}\right\rangle=\left\langle e_{1},\left[e_{2}, e_{3}\right]_{D}+\left[e_{3}, e_{2}\right]_{D}\right\rangle
\end{aligned}
$$

where $e_{1}, e_{2}, e_{3} \in \Gamma(E)$.

Regarding its application to string theory, Courant algebroids appeared in the context of generalized geometry $[13,14]$. In this case, the vector bundle is the direct sum of tangent bundle and cotangent bundle $E=T M \oplus T^{*} M$ and is introduced as an extension of the Lie algebroid of tangent vectors. We call such a Courant algebroid the standard Courant algebroid:

Definition 2.2. The standard Courant algebroid is a Courant algebroid as defined above, where we take $E=T M \oplus T^{*} M$. The anchor is the natural projection $\rho: T M \oplus T^{*} M \longrightarrow$ $T M$. The operations of the Courant algebroid are as follows:

$$
\begin{aligned}
\langle X+\alpha, Y+\beta\rangle & =\iota_{X} \beta+\iota_{Y} \alpha, \\
\rho(X+\alpha) & =X, \\
{[X+\alpha, Y+\beta]_{D} } & =[X, Y]+L_{X} \beta-\iota_{Y} d \alpha,
\end{aligned}
$$

for sections $X+\alpha, Y+\beta \in \Gamma\left(T M \oplus T^{*} M\right)$, where $X, Y$ are vector fields and $\alpha, \beta$ are 1 -forms. 
In string theory, there exists a 3 -form flux, which is usually called $H$-flux. In the case of a compactification, this flux can be nonvanishing in general. We can make a deformation of the standard Courant algebroid by a closed 3 -form $H$, which preserves the Courant algebroid conditions. We call such a deformed Courant algebroid a Courant algebroid with H-flux.

Definition 2.3. The standard Courant algebroid with H-flux is the Courant algebroid which contains the same inner product $\langle-,-\rangle$ and anchor map $\rho: T M \oplus T^{*} M \longrightarrow T M$ as the standard Courant algebroid. The Dorfman bracket is deformed by the 3-form flux to the bracket defined by

$$
[X+\alpha, Y+\beta]_{H}=[X, Y]+L_{X} \beta-\iota_{Y} d \alpha+\iota_{X} \iota_{Y} H .
$$

The Courant algebroids on $T M \oplus T^{*} M$, more precisely, exact Courant algebroids are classified by $H^{3}(M, \boldsymbol{R})[16,17]$. This means that we can only introduce the $H$-flux deformation as independent degree of freedom among all fluxes in the standard Courant algebroid.

\subsection{Supergeometric construction}

In the following, we review the supergeometric formulation of the Courant algebroids based on a so-called QP-manifold. Here, the structures of a Courant algebroid in the previous section is reconstructed by the supergeometric method.

First, we give a definition of a graded manifold. A graded manifold $\mathcal{M}$ is a ringed space, whose structure sheaf is a $\boldsymbol{Z}$-graded commutative algebra over an ordinary smooth manifold $M$. The grading is compatible with the supermanifold grading, that is, a variable of even degree is commutative and a variable of odd degree is anticommutative. By definition, the structure sheaf of $\mathcal{M}$ is locally isomorphic to $C^{\infty}(U) \otimes S^{\bullet}(V)$, where $U$ is a local chart on $M, V$ is a graded vector space, and $S^{\bullet}(V)$ is a free graded commutative ring on $V$. For rigorous mathematical definitions, we refer to $[35,36]$.

In this paper, we only consider nonnegatively graded manifolds. An $N$-manifold (i.e., a nonnegatively graded manifold) $\mathcal{M}$ equipped with a graded symplectic structure $\omega$ of degree $n$ is called P-manifold of degree $n$ and denoted by $(\mathcal{M}, \omega) . \omega$ is also called $P$-structure. We denote the degree of a function $f \in C^{\infty}(\mathcal{M})$ by $|f|$. A graded Poisson bracket on $C^{\infty}(\mathcal{M})$ is defined as $\{f, g\}=(-1)^{|f|+n+1} i_{X_{f}} i_{X_{g}} \omega$, where the Hamiltonian vector field $X_{f}$ is defined by the equation $i_{X_{f}} \omega=-\delta f$ for any $f \in C^{\infty}(\mathcal{M})$, where $\delta$ is the differential on $\mathcal{M}$. A vector field $Q$ on $\mathcal{M}$ is called homological if $Q^{2}=0$.

Definition 2.4. A QP-manifold is a P-manifold $(\mathcal{M}, \omega)$ endowed with a degree 1 homological vector field $Q$ such that $L_{Q} \omega=0$ [24].

We call the homological vector field $Q$ a $Q$-structure and the corresponding triple $(\mathcal{M}, \omega, Q)$ a QP-manifold. For any QP-manifold, there exists a Hamiltonian function $\Theta \in$ $C^{\infty}(\mathcal{M})$ associated to the homological vector field $Q$ with respect to the graded Poisson bracket $\{-,-\}$, that is,

$$
Q=\{\Theta,-\}
$$


Then the homological condition, $Q^{2}=0$, implies that $\Theta$ is a solution of the classical master equation

$$
\{\Theta, \Theta\}=0 \text {. }
$$

Such $\Theta$ is called a homological function or a Hamiltonian function. In order to construct a QP-manifold, we specify a symplectic structure $\omega$ and a Hamiltonian function $\Theta$. The classical master equation provides one unique geometric condition on the objects involved. In the next subsection, we demonstrate how the structures of a Courant algebroid emerge from a solution of the classical master equation in QP-manifold of degree 2.

\subsection{QP-manifolds of degree 2}

In this paper, we want to formulate a specific type of Courant algebroid, the Poisson Courant algebroid, using the above-mentioned supergeometric method. This formulation is possible due to the well-known equivalence of a QP-structure of degree 2 with a Courant algebroid on a vector bundle $E$ [23]. This subsection reviews the construction of the Courant algebroid on a general vector bundle $E$ including the special case where $E=$ $T M \oplus T^{*} M$. In the next section, the construction is applied to the Poisson Courant algebroid and its properties are discussed.

First, we start with a vector bundle $E$ over a smooth manifold $M$ with fiber $V$ and a graded manifold $\mathcal{M}=T^{*}[2] E[1]$. Here $E[n]$ denotes the shift of fiber degree by $n$.

Let $x^{i}$ be a local coordinate on $M$ and $e^{a}$ a basis of sections of $E$. Local coordinates on the graded double vector bundle $\mathcal{M}$ are denoted by $\left(x^{i}, \eta^{a}, \xi_{i}\right)$ with degrees $(0,1,2)$. Local coordinates on $E[1]$ are $\left(x^{i}, \eta^{a}\right)$, where $\eta^{a}$ is a local coordinate on fiber $V$ [1] of $E[1]$. By assuming a fiber metric $\langle-,-\rangle$, we identify $V^{*}[1]$ with $V[1]$. Therefore, we use the same local coordinate $\eta^{a}$ for the cotangent space $T^{*}[2]$ of the fiber of $V$ [1]. Finally, $\xi_{i}$ is a local coordinate on the fiber of $T^{*}[2] M$. The structure of the graded manifold can schematically be represented by the following diagram,

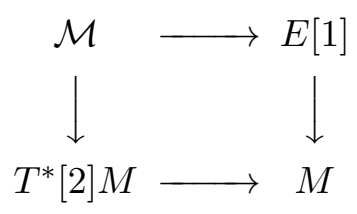

We consider the canonical embedding map of the vector bundle $E$ into $\mathcal{M}$ :

$$
j: E \oplus T M \rightarrow \mathcal{M} .
$$

The embedding map $j$ can be written using local coordinates by

$$
j:\left(x^{i}, e^{a}, \frac{\partial}{\partial x^{i}}\right) \mapsto\left(x^{i}, \eta^{a}, \xi_{i}\right) .
$$

For a section $e \in \Gamma(E)$ the pushforward is a function, $j_{*} e \in C^{\infty}(E[1])$. We use the same symbol for $E$ and $j E$, if there is no risk of confusion.

We decompose the structure sheaf by degree, i.e., the space of functions on $\mathcal{M}$ as $C^{\infty}(\mathcal{M})=\sum_{i \geq 0} C_{i}(\mathcal{M})$, where $C_{i}(\mathcal{M})$ is the space of smooth functions of degree $i$. We 
have the following equivalences by the map $j$ :

$$
\begin{aligned}
& C_{0}(\mathcal{M}) \simeq C^{\infty}(M), \\
& C_{1}(\mathcal{M}) \simeq \Gamma(E), \\
& C_{2}(\mathcal{M}) \simeq \Gamma\left(\wedge^{2} E \oplus T M\right) \\
& \text { etc. }
\end{aligned}
$$

The next step is to introduce a graded symplectic form of degree 2 on $\mathcal{M}$. We take the following symplectic structure,

$$
\omega=\delta x^{i} \wedge \delta \xi_{i}+\frac{1}{2} k_{a b} \delta \eta^{a} \wedge \delta \eta^{b}
$$

where $\left\langle\eta^{a}, \eta^{b}\right\rangle=k^{a b}$ is the fiber metric. This defines a P-structure and leads to the corresponding graded Poisson bracket $\left\{x^{i}, \xi_{j}\right\}=\delta^{i}{ }_{j}$ and $\left\{\eta^{a}, \eta^{b}\right\}=k^{a b}$.

Finally, a Q-structure on $\mathcal{M}$ is defined by a homological function $\Theta$ of degree 3 as in (2.2). Using local coordinates, the general form of a degree 3 function is given by

$$
\Theta=\rho_{a}^{i}(x) \xi_{i} \eta^{a}+\frac{1}{3 !} C_{a b c}(x) \eta^{a} \eta^{b} \eta^{c}
$$

where $\rho_{a}^{i}(x)$ and $C_{a b c}(x)$ are arbitrary local functions of $x$. The homological function satisfies the classical master equation, $\{\Theta, \Theta\}=0$. This gives a set of relations among the degree zero local functions $\rho^{i}{ }_{a}(x)$ and $C_{a b c}(x)$. The above triple $(\mathcal{M}, \omega, Q)$ defines a QP-manifold of degree 2 .

The operations on the Courant algebroid are defined using a graded Poisson bracket and derived brackets. The pseudo-metric, Dorfman bracket and anchor map are then reconstructed via the following expressions:

$$
\begin{aligned}
\left\langle e^{1}, e^{2}\right\rangle & \equiv j^{*}\left\{j_{*} e^{1}, j_{*} e^{2}\right\}, \\
{\left[e^{1}, e^{2}\right]_{D} } & \equiv j^{*}\left\{\left\{j_{*} e^{1}, \Theta\right\}, j_{*} e^{2}\right\}, \\
\rho(e) f & \equiv j^{*}\left\{j_{*} e,\left\{\Theta, j_{*} f\right\}\right\},
\end{aligned}
$$

where $f$ is a function on $M$ and $e, e^{1}, e^{2} \in \Gamma(E)$. As a consequence of the classical master equation, these three operations satisfy the defining relations of a Courant algebroid.

In the case of $E=T M \oplus T^{*} M$, we take local coordinates $\left(x^{i}, q^{i}, p_{i}, \xi_{i}\right)$ with degree $(0,1,1,2)$, where $x^{i}$ are local coordinates on $M, q^{i}$ is a local basis on the fiber of $T[1] M, p_{i}$ is a local basis on the fiber of $T^{*}[1] M$ and $\xi_{i}$ are local coordinates on the fiber of $T^{*}[2] M$. Note that we have identified $\eta^{a}=\left(q^{i}, p_{j}\right)$. The fiber metric is taken as $k=\left(\begin{array}{cc}0 & \delta^{i}{ }_{j} \\ \delta_{j}{ }^{i} & 0\end{array}\right)$. Then the graded symplectic form is given by

$$
\omega=\delta x^{i} \wedge \delta \xi_{i}+\delta q^{i} \wedge \delta p_{i} .
$$

If we take the Q-structure function as

$$
\Theta=\xi_{i} q^{i}+\frac{1}{3 !} H_{i j k}(x) q^{i} q^{j} q^{k},
$$

we get the standard Courant algebroid by (2.6). The anchor $\rho$ becomes the natural projection from $T M \oplus T^{*} M$ to $T M$ and the Dorfman bracket becomes (2.1). 


\section{Supergeometric description of Poisson Courant algebroids}

In this section, we reformulate the Poisson Courant algebroid, the Courant algebroid on a Poisson manifold in [18], using supergeometry. First, we give its definition and then construct the corresponding QP-manifold.

Definition 3.1. Let $(M, \pi)$ be a Poisson manifold with a Poisson structure $\pi \in \Gamma\left(\wedge^{2} T M\right)$ and $R \in \Gamma\left(\wedge^{3} T M\right)$ be a 3-vector field, which is closed with respect to the Poisson bivector field, $[\pi, R]_{S}=0$, where $[-,-]_{S}$ is the Schouten bracket on multivector fields on $\wedge^{\bullet} T M$.

$A$ Poisson Courant algebroid is a vector bundle $E=T M \oplus T^{*} M$ over the Poisson manifold $M$, which incorporates the three operations of a Courant algebroid. The inner product $\langle-,-\rangle$ on $T M \oplus T^{*} M$ is the same as in the standard Courant algebroid case. The bundle map $\rho: T M \oplus T^{*} M \rightarrow T M$ is defined by $\rho(X+\alpha)=\pi^{\sharp}(\alpha)$, where $\pi^{\sharp}: T^{*} M \rightarrow T M .^{2}$ The bilinear operation is defined by

$$
[X+\alpha, Y+\beta]_{R}^{\pi} \equiv[\alpha, \beta]_{\pi}+L_{\alpha}^{\pi} Y-\iota_{\beta} d_{\pi} X-\iota_{\alpha} \iota_{\beta} R,
$$

where $X+\alpha, Y+\beta \in \Gamma\left(T M \oplus T^{*} M\right), d_{\pi}(-)=[\pi,-]_{S}$ and $[-,-]_{\pi}: T^{*} M \times T^{*} M \rightarrow T^{*} M$ is the Koszul bracket given by $[\alpha, \beta]_{\pi}=L_{\pi^{\sharp}(\alpha)} \beta-L_{\pi^{\sharp}(\beta)} \alpha-d(\pi(\alpha, \beta))$. The data of a Poisson Courant algebroid can then be encoded in the quadruple $\left(E=T M \oplus T^{*} M,\langle-,-\rangle,[-,-]_{R}^{\pi}\right.$, $\left.\rho=0 \oplus \pi^{\sharp}\right)$.

We can regard the Poisson Courant algebroid as a contravariant object associated to the standard Courant algebroid. Contravariant geometry is a differential calculus in which the roles of $T M$ and $T^{*} M$ are exchanged [19,37]. Therefore, we call $[-,-]_{R}^{\pi}$ the contravariant Dorfman bracket and we can call this structure the contravariant Courant algebroid.

After giving the definition of the Poisson Courant algebroid, we reconstruct this algebroid by supergeometric methods. For this, we use the same graded manifold $\mathcal{M}=$ $T^{*}[2] T^{*}[1] M$ as in the case of the standard Courant algebroid. We also take the same symbols for the local coordinates $\left(x^{i}, q^{i}, p_{i}, \xi_{i}\right)$ and the canonical graded symplectic form (2.7).

Then the homological function defining the Q-structure for the Poisson Courant algebroid is

$$
\Theta=\pi^{i j}(x) \xi_{i} p_{j}-\frac{1}{2} \frac{\partial \pi^{j k}}{\partial x^{i}}(x) q^{i} p_{j} p_{k}+\frac{1}{3 !} R^{i j k}(x) p_{i} p_{j} p_{k},
$$

where $\pi=\frac{1}{2} \pi^{i j}(x) \frac{\partial}{\partial x^{i}} \wedge \frac{\partial}{\partial x^{j}} \in \Gamma\left(\wedge^{2} T M\right)$ is a bivector field and $R=\frac{1}{3 !} R^{i j k}(x) \frac{\partial}{\partial x^{i}} \wedge \frac{\partial}{\partial x^{j}} \wedge \frac{\partial}{\partial x^{k}}$ is a 3 -vector field. The function $\Theta$ satisfies $\{\Theta, \Theta\}=0$, i.e., it defines a Q-structure, if and only if $\pi$ is a Poisson bivector field and $R$ satisfies $[\pi, R]_{S}=0$, i.e., it is $d_{\pi}$ closed. These two conditions are exactly the ones required for the Poisson Courant algebroid.

The derived brackets define all the operations on $C^{\infty}\left(T^{*}[1] M \oplus T[1] M\right) \cong \Gamma(T M \oplus$ $\left.T^{*} M\right)$ appearing in the Poisson Courant algebroid as

$$
\begin{aligned}
\rho(X+\alpha) f(x) & =j^{*}\left\{\left\{X^{i}(x) p_{i}+\alpha_{i}(x) q^{i}, \Theta\right\}, f(x)\right\}, \\
{[X+\alpha, Y+\beta]_{R}^{\pi} } & =j^{*}\left\{\left\{X^{i}(x) p_{i}+\alpha_{i}(x) q^{i}, \Theta\right\}, Y^{j}(x) p_{j}+\beta_{j}(x) q^{j}\right\}, \\
\langle X+\alpha, Y+\beta\rangle & =j^{*}\left\{j_{*}(X+\alpha), j_{*}(Y+\beta)\right\} .
\end{aligned}
$$

\footnotetext{
${ }^{2} \pi^{\sharp}: T^{*} M \rightarrow T M$ is defined by the map $\pi^{\sharp}(\alpha)=\pi^{i j} \alpha_{i}(x) \frac{\partial}{\partial x^{j}}$ for any 1 -form, $\alpha=\alpha_{i}(x) d x^{i}$.
} 
We can confirm the above relations using local coordinates. The first equation gives

$$
\rho(X+\alpha) f(x)=j^{*}\left\{\left\{X^{i}(x) p_{i}+\alpha_{i}(x) q^{i}, \Theta\right\}, f(x)\right\}=\pi^{i j} \alpha_{i} \frac{\partial f}{\partial x^{j}}(x),
$$

and thus this is the anchor map $\rho=0 \oplus \pi^{\sharp}: T M \oplus T^{*} M \rightarrow T M$. The second equation gives

$$
\begin{aligned}
{[X+\alpha, Y+\beta]_{R}^{\pi}=} & j^{*}\left\{\left\{X^{i}(x) p_{i}+\alpha_{i}(x) q^{i}, \Theta\right\}, Y^{j}(x) p_{j}+\beta_{j}(x) q^{j}\right\} \\
= & \left(\alpha_{j} \pi^{j k} \frac{\partial Y^{i}}{\partial x^{k}}+\frac{\partial X^{i}}{\partial x^{j}} \pi^{j k} \beta_{k}-\frac{\partial X^{j}}{\partial x^{k}} \pi^{k i} \beta_{j}-\frac{\partial \alpha_{j}}{\partial x^{k}} \pi^{k i} Y^{j}\right. \\
& \left.+\frac{\partial \pi^{k i}}{\partial x^{j}} X^{j} \beta_{k}-\frac{\partial \pi^{j i}}{\partial x^{k}} \alpha_{j} Y^{k}-R^{j k i} \alpha_{j} \beta_{k}\right) j^{*} p_{i} \\
& +\left(\alpha_{j} \pi^{j k} \frac{\partial \beta_{i}}{\partial x^{k}}+\frac{\partial \alpha_{i}}{\partial x^{j}} \pi^{j k} \beta_{k}+\frac{\partial \pi^{j k}}{\partial x^{i}} \alpha_{j} \beta_{k}\right) j^{*} q^{i} \\
= & {[\alpha, \beta]_{\pi}+L_{\alpha}^{\pi} Y-\iota_{\beta} d_{\pi} X-R(\alpha, \beta,-), }
\end{aligned}
$$

and thus is the Dorfman bracket of the Poisson Courant algebroid on $T M \oplus T^{*} M$. The third equation is the same as in the case of the standard Courant algebroid.

The classical master equation, $\{\Theta, \Theta\}=0$, leads to the following conditions on these operations,

$$
\begin{aligned}
\rho\left(\left[e_{1}, e_{2}\right]_{R}^{\pi}\right) & =\left[\rho\left(e_{1}\right), \rho\left(e_{2}\right)\right], \\
{\left[e_{1},\left[e_{2}, e_{3}\right]_{R}^{\pi}\right]_{R}^{\pi} } & =\left[\left[e_{1}, e_{2}\right]_{R}^{\pi}, e_{3}\right]_{R}^{\pi}+\left[e_{2},\left[e_{1}, e_{3}\right]_{R}^{\pi}\right]_{R}^{\pi}, \\
\rho\left(e_{1}\right)\left\langle e_{2}, e_{3}\right\rangle & =\left\langle\left[e_{1}, e_{2}\right]_{R}^{\pi}, e_{3}\right\rangle+\left\langle e_{2},\left[e_{1}, e_{3}\right]_{R}^{\pi}\right\rangle, \\
\rho\left(e_{1}\right)\left\langle e_{2}, e_{3}\right\rangle & =\left\langle e_{1},\left[e_{2}, e_{3}\right]_{R}^{\pi}+\left[e_{3}, e_{2}\right]_{R}^{\pi}\right\rangle,
\end{aligned}
$$

where $e_{i} \in \Gamma\left(T M \oplus T^{*} M\right)$. These are the relations required for the Poisson Courant algebroid.

Thus, in the graded manifold method, the difference between the standard Courant algebroid and the Poisson Courant algebroid lies in the choice of the homological function. The most general homological function $\Theta$ on $E=T M \oplus T^{*} M$ possible for the Courant algebroid is

$$
\begin{aligned}
\Theta= & \tau^{i}{ }_{j}(x) \xi_{i} q^{j}+\sigma^{i j}(x) \xi_{i} p_{j}+\frac{1}{3 !} H_{i j k}(x) q^{i} q^{j} q^{k}+\frac{1}{2} F_{i j}{ }^{k}(x) q^{i} q^{j} p_{k} \\
& +\frac{1}{2} Q_{i}{ }^{j k}(x) q^{i} p_{j} p_{k}+\frac{1}{3 !} R^{i j k}(x) p_{i} p_{j} p_{k} .
\end{aligned}
$$

The classical master equation then imposes structural restrictions onto the expansion coefficients. One of the conditions for $\tau$ and $\sigma$ is $\tau^{i}{ }_{k} \sigma^{j k}+\sigma^{i k} \tau^{j}{ }_{k}=0$. The two simplest solutions are $\tau=0, \sigma \neq 0$ or $\tau \neq 0, \sigma=0$. In the standard Courant algebroid case, $\tau^{i}{ }_{j}=\delta^{i}{ }_{j}$ and $\sigma=F=Q=R=0$, and in the Poisson Courant algebroid case, $\sigma=\pi$, $Q_{i}{ }^{j k}(x)=-\frac{\partial \pi^{j k}}{\partial x^{i}}(x)$ and $\tau=H=F=0$. 


\section{Duality between $H$-flux and $R$-flux}

In this section, we study the meaning of $H$-flux geometry and $R$-flux geometry. We analyze the 'duality' transformation between two Courant algebroids in terms of supergeometry and the homological algebra. The key operation is a canonical transformation on the graded symplectic manifold (the P-manifold). This duality is a generalization of the correspondence between de Rham cohomology on differential forms and Poisson cohomology on multivector fields.

In this section, we denote the homological function $\Theta$ of the standard Courant algebroid in (2.8) as $\Theta_{H}$ and the one of the Poisson Courant algebroid in (3.1) as $\Theta_{R}$.

\subsection{Flux duality transformations as canonical transformations}

Suppose the Poisson structure $\pi$ is nondegenerate. We construct the duality transformation between the standard Courant algebroid and the Poisson Courant algebroid, which is derived from the transformation between the two homological functions $\Theta_{H}$ and $\Theta_{R}$. This leads to a duality between the standard Courant algebroid cohomology and the Poisson Courant algebroid cohomology.

First, we define a canonical transformation on a P-manifold. Let $\alpha \in C^{\infty}(\mathcal{M}) . e^{\delta_{\alpha}}$ is the exponential adjoint operation,

$$
e^{\delta_{\alpha}} f=f+\{f, \alpha\}+\frac{1}{2}\{\{f, \alpha\}, \alpha\}+\cdots
$$

for any $f \in C^{\infty}(\mathcal{M})$. If $\alpha$ is of degree $n$, this transformation preserves degree and satisfies $\left\{e^{\delta_{\alpha}} f, e^{\delta_{\alpha}} g\right\}=e^{\delta_{\alpha}}\{f, g\}$, where $f, g \in C^{\infty}(\mathcal{M})$.

Definition 4.1. For any function $\alpha$ of degree $n, e^{\delta_{\alpha}}$ is called a canonical transformation.

$e^{\delta_{\alpha}}$ is also called twisting [39].

Both the standard Courant algebroid and the Poisson Courant algebroid are realized on the same P-manifold $\left(T^{*}[2] T^{*}[1] M, \omega\right)$. Therefore, the duality transformation $T$ from $H$-flux to $R$-flux is a symplectomorphism on $T^{*}[2] T^{*}[1] M$ such that the two homological functions are mapped, $T: \Theta_{H} \mapsto \Theta_{R}$, where

$$
\begin{aligned}
& \Theta_{H}=\xi_{i} q^{i}+\frac{1}{3 !} H_{i j k}(x) q^{i} q^{j} q^{k}, \\
& \Theta_{R}=\pi^{i j}(x) \xi_{i} p_{j}-\frac{1}{2} \frac{\partial \pi^{j k}}{\partial x^{i}}(x) q^{i} p_{j} p_{k}+\frac{1}{3 !} R^{i j k}(x) p_{i} p_{j} p_{k} .
\end{aligned}
$$

We denote $\alpha_{p}=\frac{1}{2} \pi^{i j}(x) p_{i} p_{j}$ and $\alpha_{q}=\frac{1}{2} \pi_{i j}^{-1}(x) q^{i} q^{j} . \quad \alpha_{p}$ generates a so-called $\beta$ transformation and $\alpha_{q}$ generates a $b$-transformation. Note that $\alpha_{q}$ is a trivial transformation on $\Theta_{H}, e^{\delta_{\alpha_{q}}} \Theta_{H}=\Theta_{H}$, and $\alpha_{p}$ is a trivial transformation on $\Theta_{R}, e^{\delta_{\alpha_{p}}} \Theta_{R}=\Theta_{R}$, from $\left\{\Theta_{H}, \alpha_{q}\right\}=0$ and $\left\{\Theta_{R}, \alpha_{p}\right\}=0$. By direct computation, we get the relation

$$
\Theta_{R}=e^{\delta_{\alpha_{p}}} e^{-\delta_{\alpha_{q}}} e^{\delta_{\alpha_{p}}} \Theta_{H},
$$


where $R=\wedge^{3} \pi^{\sharp} H$. On the basis of the QP-manifold, this canonical transformation acts as

$$
\begin{aligned}
& e^{\delta_{\alpha_{p}}} e^{-\delta_{\alpha_{q}}} e^{\delta_{\alpha_{p}}} x^{i}=x^{i}, \\
& e^{\delta_{\alpha_{p}}} e^{-\delta_{\alpha_{q}}} e^{\delta_{\alpha_{p}}} q^{i}=\pi^{i j}(x) p_{j}, \\
& e^{\delta_{\alpha_{p}}} e^{-\delta_{\alpha_{q}} e^{\delta_{\alpha_{p}}} p_{i}}=-\pi_{i j}^{-1}(x) q^{j}, \\
& e^{\delta_{\alpha_{p}}} e^{-\delta_{\alpha_{q}}} e^{\delta_{\alpha_{p}}} \xi_{i}=\xi_{i}+\frac{\partial \pi^{j k}}{\partial x^{i}} \pi_{k l}^{-1}(x) p_{j} q^{l} .
\end{aligned}
$$

The Liouville 1-form is transformed as

$$
\vartheta=\xi_{i} \delta x^{i}-q^{i} \delta p_{i} \mapsto \xi_{i} \delta x^{i}-p_{i} \delta q^{i}=\vartheta^{\prime} .
$$

Note that $j^{*} \pi^{\sharp}(\alpha)=e^{\delta_{\alpha_{p}}} e^{-\delta_{\alpha_{q}}} e^{\delta_{\alpha_{p}}} j^{*} \alpha$ for a 1 -form $\alpha$.

The standard Courant algebroid with $H$-flux and the Poisson Courant algebroid with $R$-flux are transformed by a symplectomorphism between two QP-manifolds if $\pi$ is nondegenerate. From this observation, we understand that a flux duality is characterized by a symplectomorphism between two QP-manifolds $T:(\mathcal{M}, \omega, Q) \rightarrow\left(\mathcal{M}, \omega, Q^{\prime}\right)$. In this sense, $B$ - and $\beta$-transformations are special cases of flux duality transformations.

The flux duality transformation in (4.3) will be reinterpreted in the sigma model language on an open manifold as a change of boundary conditions in subsection 6.4.

\subsection{Two cohomologies on $T^{*}[2] T^{*}[1] M$}

$Q_{H}=\left\{\Theta_{H},-\right\}$ and $Q_{R}=\left\{\Theta_{R},-\right\}$ are two coboundary operators increasing degree by 1 on the space of functions on the QP-manifold $\mathcal{M}=T^{*}[2] T^{*}[1] M$. Expanding $C^{\infty}(\mathcal{M})=$ $\sum_{i \geq 0} C_{i}(\mathcal{M})$ by degree, $(C \bullet(\mathcal{M}), Q)$ becomes the complex for both coboundary operators, the so-called standard complex of the Courant algebroid [40], where $Q=Q_{H}$ or $Q=$ $Q_{R} \cdot\left(C \bullet(\mathcal{M}), Q_{H}\right)$ defines the standard Courant algebroid cohomology $H_{\mathrm{SCA}}^{\bullet}\left(M, Q_{H}\right)$ and $\left(C \bullet(\mathcal{M}), Q_{R}\right)$ defines the Poisson Courant algebroid cohomology $H_{\mathrm{PCA}}^{\bullet}\left(M, Q_{R}\right)$.

Both cohomologies are known cohomologies on the special subspaces. As discussed in section 2, there exists the embedding map of the Courant algebroid to a graded manifold, $j: E \oplus T M \rightarrow T^{*}[2] T^{*}[1] M$, where $E=T M \oplus T^{*} M$ is the Courant algebroid. We take a local basis $\left(\frac{\partial}{\partial x^{i}}, d x^{i}, \frac{\partial}{\partial x^{i}}\right)$ on $E \oplus T M .^{3}$ The local basis is mapped as $j:\left(\frac{\partial}{\partial x^{i}}, d x^{i}, \frac{\partial}{\partial x^{i}}\right) \mapsto$ $\left(p_{i}, q^{i}, \xi_{i}\right)$.

First, we consider the standard Courant algebroid with H-flux. Since $T[1] M$ is isomorphic to $T^{*} M$, let us consider the subspace $C^{\infty}(T[1] M)$. An element $\gamma \in C^{\infty}(T[1] M)$ can be written ss

$$
\gamma=\frac{1}{s !} \gamma_{i_{1} \cdots i_{s}}(x) q^{i_{1}} \cdots q^{i_{s}} .
$$

$\gamma$ is mapped to a differential form by pullback,

$$
j^{*} \gamma=\frac{1}{s !} \gamma_{i_{1} \cdots i_{s}}(x) d x^{i_{1}} \wedge \cdots \wedge d x^{i_{s}} .
$$

\footnotetext{
${ }^{3}$ The first $\frac{\partial}{\partial x^{i}}$ is the basis of the tangent bundle in the Courant algebroid and the third $\frac{\partial}{\partial x^{i}}$ is the basis of the tangent bundle of the image of the anchor map.
} 
Thus, $C^{\infty}(T[1] M)$ is equivalent to the space of differential forms $\Omega^{\bullet}(M)$. We can easily show that the operation of $Q_{H}$ on $C^{\infty}(T[1] M)$ is the de Rham differential $d$ on $\Omega^{\bullet}(M)$,

$$
d\left(j^{*} \gamma\right)=-j^{*} Q_{H} \gamma
$$

Therefore, the restriction of the standard Courant cohomology $H_{\mathrm{SCA}}^{\bullet}\left(M, Q_{H}\right)$ to $C^{\infty}(T[1] M)$ is equivalent to the de Rham cohomology,

$$
\left.H_{\mathrm{SCA}}^{\bullet}\left(M, Q_{H}\right)\right|_{C^{\infty}(T[1] M)} \simeq H_{d R}^{\bullet}(M, d) .
$$

Next, we consider the Poisson Courant algebroid case. Note that $T^{*}[1] M$ is isomorphic to $T M$. Let us consider the subspace $C^{\infty}\left(T^{*}[1] M\right)$, whose elements can be written as

$$
u=\frac{1}{s !} u^{i_{1} \cdots i_{s}}(x) p_{i_{1}} \cdots p_{i_{s}} .
$$

The pullback maps $u$ to a multivector field,

$$
j^{*} u=\frac{1}{s !} u^{i_{1} \cdots i_{s}}(x) \frac{\partial}{\partial x^{i_{1}}} \wedge \cdots \wedge \frac{\partial}{\partial x^{i_{s}}} .
$$

Hence, $C^{\infty}\left(T^{*}[1] M\right)$ is equivalent to the space of multivector fields $T_{\text {poly }}^{\bullet}(M)$.

If we put $R=0$, then $\Theta_{R}$ is given by

$$
\left.\Theta_{R}\right|_{R=0}=-\left\{\pi, \Theta_{0}\right\}=-\left\{\frac{1}{2} \pi^{j k}(x) p_{j} p_{k}, \xi_{i} q^{i}\right\}
$$

and

$$
Q_{R} u=\left\{\Theta_{R}, u\right\}=-\left\{\left\{\pi, \Theta_{0}\right\}, u\right\},
$$

for $u \in C^{\infty}\left(T^{*}[1] M\right)$. Since the derived bracket $\left\{\left\{-, \Theta_{0}\right\},-\right\}$ is equivalent to the Schouten bracket $[-,-]_{S}$, and the graded Poisson bracket of $R$ with elements of $C^{\infty}\left(T^{*}[1] M\right)$ is zero, $Q_{R}$ is equivalent to the Poisson differential $d_{\pi}=[\pi,-]_{S}$ on $T_{\text {poly }}^{\bullet}(M)$,

$$
d_{\pi}\left(j^{*} u\right)=-j^{*} Q_{R} u
$$

The cohomology defined by the coboundary operator $d_{\pi}$ is the Poisson cohomology, $H_{P}^{k}\left(M, d_{\pi}\right)$. Therefore, the restriction of the Poisson Courant cohomology $H_{\mathrm{PCA}}^{\bullet}\left(M, Q_{R}\right)$ to $C^{\infty}\left(T^{*}[1] M\right)$ is equivalent to the Poisson cohomology,

$$
\left.H_{\mathrm{PCA}}^{\bullet}\left(M, Q_{R}\right)\right|_{C^{\infty}\left(T^{*}[1] M\right)} \simeq H_{P}^{\bullet}\left(M, d_{\pi}\right) .
$$

\subsection{Duality of cohomologies}

Since we are considering an even dimensional manifold and a nondegenerate Poisson structure, we can prove that $H_{\mathrm{SCA}}^{\bullet}\left(M, Q_{H}\right)$ is isomorphic to $H_{\mathrm{PCA}}^{\bullet}\left(M, Q_{R}\right)$. This is a generalization of the well known result that if $M$ is symplectic, the de Rham cohomology and the Poisson cohomology are isomorphic, $H_{d R}^{k}(M, d) \simeq H_{P}^{k}\left(M, d_{\pi}\right)[37]$. 
The map $\wedge^{k} \pi^{\sharp}: \Omega^{k}(M) \rightarrow T_{\text {poly }}^{k}(M)$ induces a homomorphism between de Rham cohomology and Poisson cohomology,

$$
\wedge^{k} \pi^{\sharp}: H_{d R}^{k}(M, d) \rightarrow H_{P}^{k}\left(M, d_{\pi}\right) .
$$

If $\pi^{-1}$ is symplectic, the de Rham cohomology on $\Omega^{\bullet}(M)$ is isomorphic to the Poisson cohomology:

$$
H_{d R}^{k}(M, d) \simeq H_{P}^{k}\left(M, d_{\pi}\right)
$$

In our duality theory, the de Rham cohomology is extended to $H_{\mathrm{SCA}}^{\bullet}\left(M, Q_{H}\right)$ and the Poisson cohomology is extended to $H_{\mathrm{PCA}}^{\bullet}\left(M, Q_{R}\right)$. Duality of $\mathrm{H}$-flux and R-flux is understood as duality of $H_{\mathrm{SCA}}^{\bullet}\left(M, Q_{H}\right)$ and $H_{\mathrm{PCA}}^{\bullet}\left(M, Q_{R}\right)$ on the same space $C^{\infty}(\mathcal{M})$.

Let $f$ be a general function on $\mathcal{M}=T^{*}[2] T^{*}[1] M, f \in C^{\infty}(\mathcal{M})$. From (4.3), it follows that $Q_{H} f=0 \Leftrightarrow Q_{R}\left(e^{\delta_{\alpha_{p}}} e^{-\delta_{\alpha_{q}}} e^{\delta_{\alpha_{p}}} f\right)=0$ and $f=Q_{H} g \Leftrightarrow f=$ $Q_{R}\left(e^{\delta_{\alpha_{p}}} e^{-\delta_{\alpha_{q}}} e^{\delta_{\alpha_{p}}} g\right)$. Therefore, we obtain maps from elements of the $Q_{H^{-}}$complex to elements of the $Q_{R}$-complex,

$$
T: f \mapsto e^{\delta_{\alpha_{p}}} e^{-\delta_{\alpha_{q}}} e^{\delta_{\alpha_{p}}} f .
$$

The flux duality map of complexes $T: C_{\bullet}(\mathcal{M}) \rightarrow C_{\bullet}(\mathcal{M})$ gives rise to the isomorphism of cohomologies, $T: H_{\mathrm{SCA}}^{\bullet}\left(M, d_{H}\right) \rightarrow H_{\mathrm{PCA}}^{\bullet}\left(M, d_{R}\right)$.

We obtain the following theorem.

Theorem 4.2. Let $\pi$ be a nondegenerate Poisson structure, that is, $\pi^{-1}$ is symplectic, and $R=\wedge^{3} \pi^{\sharp} H$. Then, the standard Courant algebroid cohomology is isomorphic to the Poisson Courant algebroid cohomology,

$$
H_{\mathrm{SCA}}^{k}\left(M, Q_{H}\right) \stackrel{\sim}{\rightarrow} H_{\mathrm{PCA}}^{k}\left(M, Q_{R}\right) .
$$

\section{Poisson Courant algebroids from double field theory}

In this section, we show that the Poisson Courant algebroid is a solution of the section condition (the strong constraint) in double field theory. This shows that the Poisson Courant algebroid is directly connected to the geometry of double field theory.

\subsection{Supergeometric formulation, Poisson structure and double field theory}

We start with the supergeometric formulation of the geometry of double field theory [38]. We take a doubled configuration space $\widehat{M}$ in $2 d$ dimensions with local coordinates $\left(y^{i}, \tilde{y}_{i}\right)$ and a QP-manifold of degree $2, T^{*}[2] \widehat{M}$ with fiber coordinates, $\left(\eta_{i}, \tilde{\eta}^{i}\right)$, such that $\left\{y^{i}, \eta_{j}\right\}=$ $\left\{\tilde{y}_{j}, \tilde{\eta}^{i}\right\}=\delta^{i}{ }_{j}$. Moreover, we introduce degree one canonical conjugate coordinates $\left(q^{i}, p_{i}\right)$ such that $\left\{q^{i}, p_{j}\right\}=\delta^{i}{ }_{j}$.

On this P-manifold, the geometry of double field theory is formulated using the Qstructure homological function,

$$
\Theta_{C}=\eta_{i} q^{i}+\tilde{\eta}^{i} p_{i}
$$


The classical master equation, $\left\{\Theta_{C}, \Theta_{C}\right\}=0$, gives rise to the section condition,

$$
\tilde{\eta}^{i} \eta_{i}=0 .
$$

The C-bracket is constructed by the derived bracket,

$$
\left[e_{1}, e_{2}\right]_{C}=j^{*}\left\{\left\{j_{*} e_{1}, \Theta_{C}\right\}, j_{*} e_{2}\right\},
$$

where $e_{1}, e_{2}$ are sections of $T \widehat{M} \oplus T^{*} \widehat{M}$.

We choose a nontrivial physical configuration space, a $d$-dimensional submanifold $M \subset$ $\widehat{M}$ with local coordinate $x^{i}$ under the assumption that $M$ has a Poisson structure $\pi$. We can consider a local coordinate transformation with following Jacobian,

$$
\frac{\partial(x, \tilde{x})}{\partial(y, \tilde{y})}=\left(\begin{array}{ll}
\frac{\partial x^{i}}{\partial y^{j}} & \frac{\partial x^{i}}{\partial \tilde{y}_{j}} \\
\frac{\partial \tilde{x}_{i}}{\partial y^{j}} & \frac{\partial \tilde{x}_{i}}{\partial \tilde{y}_{j}}
\end{array}\right)=\left(\begin{array}{cc}
\delta^{i}{ }_{j} & \pi^{i j} \\
0 & \delta_{i}{ }^{j}
\end{array}\right) .
$$

Alternatively, this local coordinate transformation can be realized as a twist of the original $\Theta_{C}$ by the canonical function $\alpha_{p}=\frac{1}{2} \pi^{i j}(x) p_{i} p_{j}$. Here, we denote the original homological function as $\Theta_{C}^{\prime}=\xi_{i} q^{i}+\tilde{\xi}^{i} p_{i}$. The canonical transformation deforms the homological function,

$$
\Theta_{C}=e^{\alpha_{p}} \Theta_{C}^{\prime}=\xi_{i} q^{i}+\tilde{\xi}^{i} p_{i}+\pi^{i j} \xi_{i} p_{j}-\frac{1}{2} \frac{\partial \pi^{j k}}{\partial x^{i}}(x) q^{i} p_{j} p_{k} .
$$

This corresponds to the change of variables,

$$
\begin{aligned}
\eta_{i} & =\xi_{i}-\frac{1}{2} \frac{\partial \pi^{j k}}{\partial x^{i}}(x) p_{j} p_{k}, \\
\tilde{\eta}^{i} & =\tilde{\xi}^{i}-\pi^{i j} \xi_{j} .
\end{aligned}
$$

We need the second term in (5.6) for consistency of the Poisson structure with the local coordinate transformation (5.4). The section condition is deformed to

$$
\tilde{\xi}^{i}\left(\xi_{i}-\frac{1}{2} \frac{\partial \pi^{j k}}{\partial x^{i}}(x) p_{j} p_{k}\right)=0 .
$$

Finally, we take $\tilde{\xi}^{i}=0$ corresponding to the submanifold defined by $\tilde{x}_{i}=0$, and obtain homological functions of both standard and Poisson Courant algebroids,

$$
\left.\Theta_{C}\right|_{\tilde{x}=0}=\Theta_{H=0}+\Theta_{R=0} .
$$

Note that $\Theta_{C}$ defines a double complex, since $\left\{\Theta_{H=0}, \Theta_{R=0}\right\}=0$. In this paper, we analyze these two Courant algebroids. In fact, $\Theta_{H=0}+\Theta_{R=0}$ defines a Lie bialgebroid on $T M \oplus T^{*} M{ }^{4}$ Since we change the section condition $\tilde{\eta}^{i}=0$ to $\tilde{\xi}^{i}=0$, in general, the configuration space $M$ is not embedded as a direct product $M \times \tilde{M}$ in the doubled space, but is a nontrivial submanifold of the doubled configuration space.

\footnotetext{
${ }^{4}$ In the case where $H$ and $R$ are nonzero this defines a proto-Lie bialgebroid [34, 42]. Solutions of the classical master equation give a dependency between both fluxes if the Courant algebroid is exact [16, 17].
} 


\subsection{Poisson Courant algebroid $R$-flux in double field theory}

Finally, we want to discuss how the $R$-flux of the Poisson Courant algebroid relates to the $R$-flux in double field theory. For this, we compute the $B$ - and $\beta$-twist of the homological function (5.1). This gives the following description of all fluxes $H, F, Q$ and $R$ in terms of their potentials

$$
\begin{aligned}
e^{-\delta_{\beta}} e^{-} \delta_{B} & \Theta_{C}=\left(\eta_{i}-B_{m i} \tilde{\eta}^{m}\right) q^{i}+\left(\tilde{\eta}^{i}-\eta_{m} \beta^{m i}+\tilde{\eta}^{n} B_{n m} \beta^{m i}\right) p_{i} \\
+ & \frac{1}{2}\left[-B_{i n} \tilde{\partial}^{i} B_{r s}+\partial_{n} B_{r s}\right] q^{n} q^{r} q^{s} \\
+ & {\left[\frac{1}{2} \tilde{\partial}^{i} B_{m n}+\left(B_{l m} \tilde{\partial}^{l} B_{n s}-\partial_{m} B_{n s}+\frac{1}{2} B_{l s} \tilde{\partial}^{l} B_{m n}-\frac{1}{2} \partial_{s} B_{m n}\right) \beta^{s i}\right] p_{i} q^{m} q^{n} } \\
+ & {\left[\frac{1}{2} \partial_{i} \beta^{h k}-\frac{1}{2} B_{l i} \tilde{\partial}^{l} \beta^{h k}+\tilde{\partial}^{h} B_{i n} \beta^{n k}\right.} \\
& \left.-\frac{1}{2}\left[-B_{l i} \tilde{\partial}^{l} B_{r s}+\partial_{i} B_{r s}-B_{l s} \tilde{\partial}^{l} B_{i r}+\partial_{s} B_{i r}+B_{l r} \tilde{\partial}^{l} B_{i s}-\partial_{r} B_{i s}\right] \beta^{s h} \beta^{r k}\right] q^{i} p_{h} p_{k} \\
+ & {\left[\frac{1}{2} \tilde{\partial}^{i} \beta^{h k}-\frac{1}{4} \partial_{l} \beta^{i h} \beta^{l k}-\frac{1}{4} \beta^{l i} \partial_{l} \beta^{h k}+\frac{1}{4} B_{l n} \tilde{\partial}^{l} \beta^{i h} \beta^{n k}\right.} \\
+ & \frac{1}{4} B_{l n} \beta^{n i} \tilde{\partial}^{l} \beta^{h k}-\frac{1}{2} \tilde{\partial}^{i} B_{m n} \beta^{n h} \beta^{m k} \\
+ & \left.\frac{1}{3 !}\left(-B_{l n} \tilde{\partial}^{l} B_{r s}+\partial_{n} B_{r s}-B_{l s} \tilde{\partial}^{l} B_{n r}+\partial_{s} B_{n r}+B_{l r} \tilde{\partial}^{l} B_{n s}-\partial_{r} B_{n s}\right) \beta^{s i} \beta^{r h} \beta^{n k}\right] p_{i} p_{h} p_{k},
\end{aligned}
$$

where $B=\frac{1}{2} B_{i j}(y, \tilde{y}) q^{i} q^{j}$ and $\beta=\frac{1}{2} \beta^{i j}(y, \tilde{y}) p_{i} p_{j}$ are functions on the ordinary coordinates $y$ and their duals $\tilde{y}$. The Q-structure function of the $H$-twisted standard Courant algebroid (2.8) realizes the double field theory $H$-flux in the supergravity limit $\left(\tilde{\eta}^{i}=\tilde{\partial}^{i}=0\right)$ with vanishing $\beta$-field, $\beta^{i j}=0$. Indeed, truncation of (5.10) to this frame leads to the correct local description of $H$-flux in terms of its potential $H_{n r s}=\frac{1}{2} \partial_{[n} B_{r s]}$. On the other hand, truncation to the non-geometric frame, where $\eta_{i}=\partial_{i}=0$ and $B_{i j}=0$, leads to the correct description of $R$-flux in terms of its potential $R^{i h k}=\frac{1}{2} \tilde{\partial}^{[i} \beta^{h k]}$. Finally, the truncation to the frame such that $\tilde{\eta}^{i}=\tilde{\partial}^{i}=0$ and $B_{i j}=0$ gives the correct description of $R$-flux in terms of $R^{i h k}=\frac{1}{2} \beta^{[i|l|} \partial_{l} \beta^{h k]}$. This brings us into the position to compare the $R$-flux of the Poisson Courant algebroid to the $R$-flux of double field theory. For this, we have to distinguish two cases, which will be discussed in the following.

The first case concerns the meaning of the transformation $\Theta_{H} \rightarrow \Theta_{R}$ (4.3) of the standard Courant algebroid with $H$-flux to the Poisson Courant algebroid $R$-flux. For this transformation, we introduce the Poisson bivector field $\pi$. Since, as described above, the standard Courant algebroid with $H$-flux already works in the supergravity frame with zero $\beta$-field by the identification $y^{i}=x^{i}$ and $\tilde{y}_{i}=\tilde{x}_{i}=0$, the resulting Poisson Courant algebroid also works in the same frame. The Poisson tensor $\pi$ is introduced as additional freedom, which is not related to the flux potentials $\beta$ and $B$, and the resulting Poisson Courant algebroid with $R$-flux serves as a different way of representing an $H$-flux background on a Poisson manifold. On the other hand, the term $-\frac{1}{2} \frac{\partial \pi^{i j}}{\partial x^{k}} q^{k} p_{i} p_{j}$ in (3.1) is a so-called Poisson 
connection with vanishing curvature. Therefore, it must be distinguished from a $Q$-flux term in double field theory.

In the second case, if the Poisson Courant algebroid is seen as a stand-alone object, we can make contact to the double field theory $R$-flux. Through identification of (5.10) to (3.1) we find

$$
\begin{aligned}
\pi^{i j}(x) \xi_{i} & =\tilde{\eta}^{j}-\eta_{m} \beta^{m j}(y, \tilde{y})+\tilde{\eta}^{n} B_{n m}(y, \tilde{y}) \beta^{m j}(y, \tilde{y}), \\
0 & =\eta_{i}-B_{m i}(y, \tilde{y}) \tilde{\eta}^{m}
\end{aligned}
$$

which leads to the identification $\pi^{i j}(x) \xi_{i}=\tilde{\eta}^{j}$ or $\pi^{i j}(x) \frac{\partial}{\partial x^{i}}=\frac{\partial}{\partial \tilde{y}_{j}}$ and we can read off how the section condition is solved. Integration of this equation leads to

$$
\tilde{y}_{i}=\int \pi_{i j}^{-1}(x) d x^{j}
$$

Since there is no $H$-flux coefficient in the Poisson Courant algebroid, we obtain the relation $B_{i j}=0$, which leads to $\frac{\partial}{\partial y^{i}}=0$ due to (5.12). The term $-\frac{1}{2} \frac{\partial \pi^{i j}}{\partial x^{k}} q^{k} p_{i} p_{j}$ in (3.1) is not sourced by the potentials $B$ or $\beta$, but is a Poisson connection arising from the underlying space, and its origin is different from the $Q$-flux in double field theory. Finally, the local description of $R$-flux is then given in terms of the $\beta$-potential via

$$
\begin{aligned}
R^{i h k} & =\left.\frac{1}{2} \frac{\partial}{\partial \tilde{y}_{[i}} \beta^{h k]}(y, \tilde{y})\right|_{y=0, \tilde{y}=\int \pi^{-1}(x) d x} \\
& =\frac{1}{2} \pi^{j[i}(x) \frac{\partial}{\partial x^{j}} \beta^{h k]}(x) \\
& =\frac{1}{2}[\pi, \beta]_{S} .
\end{aligned}
$$

To summarize, the Poisson Courant algebroid can be interpreted in two different ways, depending on the frame chosen in double field theory. In order to analyze the property of $R$-flux, we can use this correspondence, and on spacetime with a Poisson structure, some parts of $R$-flux geometry can be analyzed as $H$-flux geometry.

\section{Topological sigma models}

We want to consider field theoretical models with Poisson Courant algebroid symmetry. Here, we construct a 3-dimensional AKSZ sigma model, i.e., a theory of a topological membrane with 3 -vector flux $R$, following the construction of a topological membrane theory based on the standard Courant algebroid [41, 42]. For this purpose, first we shortly review the concept of AKSZ sigma models [26, 30, 43].

\subsection{AKSZ sigma models}

Let $(\mathcal{X}, D, \mu)$ be a differential graded manifold $\mathcal{X}$ with a $D$-invariant nondegenerate measure $\mu$, where $D$ is a differential on $\mathcal{X}$. Let $(\mathcal{M}, \omega, Q)$ be a $\mathrm{QP}$-manifold, and let $\operatorname{Map}(\mathcal{X}, \mathcal{M})$ be the space of smooth maps from $\mathcal{X}$ to $\mathcal{M}$. It means that we consider the sigma model on the worldvolume $\mathcal{X}$ embedded into the target space $\mathcal{M}$. 
Since $\operatorname{Diff}(\mathcal{X}) \times \operatorname{Diff}(\mathcal{M})$ naturally acts on $\operatorname{Map}(\mathcal{X}, \mathcal{M}), D$ and $Q$ induce differentials $\hat{D}$ and $\check{Q}$, respectively, on $\operatorname{Map}(\mathcal{X}, \mathcal{M})$. Explicitly, $\hat{D}(z, f)=D(z) \delta f(z)$ and $\check{Q}(z, f)=Q f(z)$, for all $z \in \mathcal{X}$ and $f \in \operatorname{Map}(\mathcal{X}, \mathcal{M})$.

The evaluation map ev $: \mathcal{X} \times \operatorname{Map}(\mathcal{X}, \mathcal{M}) \longrightarrow \mathcal{M}$ is defined as ev $:(z, f) \longmapsto f(z)$, for any $z \in \mathcal{X}$ and $f \in \operatorname{Map}(\mathcal{X}, \mathcal{M})$. The chain map on the space of graded differential forms, $\mu_{*}: \Omega^{\bullet}(\mathcal{X} \times \operatorname{Map}(\mathcal{X}, \mathcal{M})) \longrightarrow \Omega^{\bullet}(\operatorname{Map}(\mathcal{X}, \mathcal{M}))$, is defined as

$$
\mu_{*} \omega(f)\left(v_{1}, \ldots, v_{k}\right)=\int_{\mathcal{X}} \mu(z) \omega(z, f)\left(v_{1}, \ldots, v_{k}\right)
$$

for a graded differential form $\omega$, where $v_{i}$ are a vector fields on $\mathcal{X}$ and $\int_{\mathcal{X}} \mu$ is the Berezin integration on $\mathcal{X}$. The composition $\mu_{*} \mathrm{ev}^{*}: \Omega^{\bullet}(\mathcal{M}) \longrightarrow \Omega^{\bullet}(\operatorname{Map}(\mathcal{X}, \mathcal{M}))$ is called transgression map.

A P-structure $\boldsymbol{\omega}$ on $\operatorname{Map}(\mathcal{X}, \mathcal{M})$ is defined by

$$
\boldsymbol{\omega}:=\mu_{*} \mathrm{ev}^{*} \omega
$$

Note that $\boldsymbol{\omega}$ is nondegenerate and closed since the operation $\mu_{*} \mathrm{ev}^{*}$ preserves these properties. The corresponding graded Poisson bracket on the mapping $\operatorname{space} \operatorname{Map}(\mathcal{X}, \mathcal{M})$ is also denoted by $\{-,-\}$. We show later that this bracket is the BV bracket $\{-,-\}_{\mathrm{BV}}$ or the Poisson bracket $\{-,-\}_{\mathrm{PB}}$.

A $Q$-structure function $S$ on $\operatorname{Map}(\mathcal{X}, \mathcal{M})$ is constructed as follows. $S$ consists of two parts $S=S_{0}+S_{1}$. We take a canonical 1-form (the Liouville 1-form) $\vartheta$ on $\mathcal{M}$ such that $\omega=-\delta \vartheta$ and define $S_{0}=\iota^{\iota} \mu_{*} \mathrm{ev}^{*} \vartheta$. Moreover, we define $S_{1}=\mu_{*} \mathrm{ev}{ }^{*} \Theta$, where $\Theta$ is the homological function on $\mathcal{M}$. Then we can prove that $S$ is a homological function on $\operatorname{Map}(\mathcal{X}, \mathcal{M})$

$$
\{S, S\}=0
$$

using the definitions of $S_{0}$ and $S_{1}$ and the properties of the maps. A degree 1 homological vector field $\boldsymbol{Q}$ is defined as $\boldsymbol{Q}=\{S,-\}$. The classical master equation shows that $\boldsymbol{Q}$ is a coboundary operator, $\boldsymbol{Q}^{2}=0$.

From the above construction, we can prove that the mapping $\operatorname{space} \operatorname{Map}(\mathcal{X}, \mathcal{M})$ is a QP-manifold. This structure is called an AKSZ sigma model. If $\mathcal{X}=T[1] X$, where $X$ is a manifold in $n+1$ dimensions, the $\mathrm{QP}$-structure on $\operatorname{Map}(\mathcal{X}, \mathcal{M})$ is of degree -1 . In this case, a QP-structure on $\operatorname{Map}(T[1] X, \mathcal{M})$ is equivalent to the Batalin-Vilkovisky formalism of a topological sigma model including all ghosts and antifields. $\{-,-\}$ is the $\mathrm{BV}$ antibracket, and it is denoted by $\{-,-\}_{\mathrm{BV}}$.

If $X$ is a manifold in $n$ dimensions, the $\mathrm{QP}$-structure on $\operatorname{Map}(\mathcal{X}, \mathcal{M})$ is of degree 0 . In this case, a $\mathrm{QP}$-structure on $\operatorname{Map}(T[1] X, \mathcal{M})$ is equivalent to the Hamiltonian $\mathrm{BFV}$ formalism and $\{-,-\}$ is an ordinary Poisson bracket. Then we denote $\{-,-\}$ by $\{-,-\}_{\mathrm{PB}}$.

\subsection{AKSZ sigma models with boundary}

Recall the definition of a canonical transformation in subsection 4.1. Let $\mathcal{M}$ be a QPmanifold of degree $n$. For any function $\alpha \in C^{\infty}(\mathcal{M})$ of degree $n, e^{\delta_{\alpha}}$ is called a canonical transformation, since $\left\{e^{\delta_{\alpha}} f, e^{\delta_{\alpha}} g\right\}=e^{\delta_{\alpha}}\{f, g\}$, where $f, g \in C^{\infty}(\mathcal{M})$. 
For our purpose, we choose a special kind of canonical transformation, called canonical function with respect to a Lagrangian submanifold $\mathcal{L}$.

Definition 6.1. Let $(\mathcal{M}, \omega, Q)$ be a QP-manifold of degree $n$. A function $\alpha$ of degree $n$ is called canonical function with respect to $\mathcal{L}$, if $\left.e^{\delta_{\alpha}} \Theta\right|_{\mathcal{L}}=0$, where $\mathcal{L}$ is a Lagrangian submanifold of $(\mathcal{M}, \omega)$ and $\left.\right|_{\mathcal{L}}$ is the restriction to $\mathcal{L}$.

This transformation changes the target QP-manifold to $\left(\mathcal{M}, \omega, \Theta_{\alpha}\right)$ with $\Theta_{\alpha}=e^{\delta_{\alpha}} \Theta$. Since the P-structure does not change, the new Q-structure function $S^{\prime}$ in the AKSZ sigma model becomes

$$
\begin{aligned}
S^{\prime} & =S_{0}+S_{1}^{\prime} \\
& =\iota_{\hat{D}} \mu_{*} \mathrm{ev}^{*} \vartheta+\mu_{*} \mathrm{ev}^{*} e^{\delta_{\alpha}} \Theta .
\end{aligned}
$$

If $\partial X=\emptyset, S^{\prime}$ also satisfies the classical master equation, since $\left\{e^{\delta_{\alpha}} \Theta, e^{\delta_{\alpha}} \Theta\right\}=e^{\delta_{\alpha}}\{\Theta, \Theta\}=$ 0 . If $\partial X \neq \emptyset$, the boundary conditions are deformed by $\alpha$, so that $S^{\prime}$ satisfies the classical master equation. In this case, using Stokes' theorem, a straightforward computation gives

$$
\left\{S^{\prime}, S^{\prime}\right\}=\iota_{\hat{D}} \mu_{\partial \mathcal{X}_{*}}\left(i_{\partial} \times \mathrm{id}\right)^{*} \mathrm{ev}^{*} \vartheta+\mu_{\partial \mathcal{X} *}\left(i_{\partial} \times \mathrm{id}\right)^{*} \mathrm{ev}^{*} e^{\delta_{\alpha}} \Theta
$$

where $i_{\partial}$ is the inclusion map $i_{\partial}: \partial \mathcal{X} \longrightarrow \mathcal{X}$ and $\mu_{\partial \mathcal{X} *}$ is the boundary integration on $\partial \mathcal{X}$ by the pullback $\mu_{*}$ by the map $i_{\partial}$. Since the classical master equation, $\left\{S^{\prime}, S^{\prime}\right\}=0$, must be satisfied for consistency of the theory, the right hand side of (6.3) must vanish. The condition is expressed on $\mathcal{M}$ as follows [29, 44].

Proposition 6.2. We assume $\partial \mathcal{X} \neq \emptyset$. Let $(\mathcal{M}, \omega, \Theta)$ be a QP-manifold of degree $n$ and $\mathcal{L}$ a Lagrangian submanifold of $\mathcal{M}$, which is the zero locus of the canonical 1-form $\vartheta$, where $\omega=-\delta \vartheta$.

Let $\alpha \in C^{\infty}(\mathcal{M})$ of degree $n$ be a canonical function with respect to $\mathcal{L}$, i.e., $\left.e^{\delta_{\alpha}} \Theta\right|_{\mathcal{L}}=0$. Then, the classical master equation, $\left\{S^{\prime}, S^{\prime}\right\}=0$, is satisfied in an $A K S Z$ sigma model (6.2).

From proposition 6.2, the mathematical structure of an AKSZ sigma model with boundary is described by a quintuple $(\mathcal{M}, \omega, \Theta, \mathcal{L}, \alpha)$.

A canonical transformation by a canonical function $\alpha$ can be interpreted as an introduction of a boundary term in the sigma model action $S$.

Let $S^{\prime \prime}=e^{-\delta_{\hat{\alpha}}} S^{\prime}$, where $\hat{\alpha}=\mu_{*} \mathrm{ev}^{*} \alpha$. Then, $S^{\prime}$ and $S^{\prime \prime}$ have equivalent geometric structures,

$$
\begin{aligned}
S^{\prime \prime} & =e^{-\delta_{\hat{\alpha}}} S^{\prime} \\
& =e^{-\delta_{\hat{\alpha}}} S_{0}+\mu_{*} \mathrm{ev}^{*} e^{-\delta_{\alpha}} e^{\delta_{\alpha}} \Theta \\
& =e^{-\delta_{\hat{\alpha}}} S_{0}+\mu_{*} \mathrm{ev}^{*} \Theta .
\end{aligned}
$$

Let us consider the special case, where $\alpha$ satisfies $\{\alpha, \alpha\}=0$. Then, since $e^{-\delta_{\hat{\alpha}}} S_{0}=$ $S_{0}-\left\{S_{0}, \hat{\alpha}\right\}, \alpha$ generates a boundary term $S_{\partial \mathcal{X}}=-\mu_{\partial \mathcal{X} *}\left(i_{\partial} \times \mathrm{id}\right)^{*} \operatorname{ev}^{*} \alpha$,

$$
\begin{aligned}
S^{\prime \prime} & =S_{0}-\left\{S_{0}, \hat{\alpha}\right\}+\mu_{*} \mathrm{ev}^{*} \Theta \\
& =S_{0}-L_{\hat{D}} \mu_{*} \mathrm{ev}^{*} \alpha+\mu_{*} \mathrm{ev}^{*} \Theta \\
& =S-\mu_{\partial \mathcal{X} *}\left(i_{\partial} \times \mathrm{id}\right)^{*} \mathrm{ev}^{*} \alpha .
\end{aligned}
$$


Therefore, twisting of $S$ by $\alpha$ introduces a boundary term induced by transgression of $\alpha$, $S_{\partial \mathcal{X}}=-\int_{\partial \mathcal{X}} \mu\left(i_{\partial} \times \mathrm{id}\right)^{*} \mathrm{ev}^{*} \alpha$.

\subsection{Contravariant Courant sigma models}

We construct the AKSZ sigma model induced from the Poisson Courant algebroid.

Let us take a 3-dimensional manifold $X$ with boundary $\partial X$. The worldvolume is a supermanifold $\mathcal{X}=T[1] X$. Let $\left(\sigma^{\mu}, \theta^{\mu}\right)$ be local coordinates of degree $(0,1)$ on $\mathcal{X}$. Elements of $\operatorname{Map}(\mathcal{X}, \mathcal{M})$ are superfields, which we denote by boldface letters. For example, $\boldsymbol{e} \in \Gamma\left(\mathcal{X}, \boldsymbol{x}^{*} \mathcal{M}\right)$ corresponds to a local coordinate $e$ on $\mathcal{M}$, where $\boldsymbol{x}: \mathcal{X} \rightarrow M$.

The AKSZ construction on $\operatorname{Map}\left(\mathcal{X}, T^{*}[2] T^{*}[1] M\right)$ gives the bulk AKSZ sigma model. We denote the P-structure by

$$
\boldsymbol{\omega}=\int_{\mathcal{X}} \mu\left(\delta \boldsymbol{x}^{i} \wedge \delta \boldsymbol{\xi}_{i}+\delta \boldsymbol{q}^{i} \wedge \delta \boldsymbol{p}_{i}\right)
$$

If $\alpha=0$, the $\mathrm{Q}$-structure function has the following form:

$$
S=\int_{\mathcal{X}} \mu\left(\boldsymbol{\xi}_{i} \boldsymbol{d} \boldsymbol{x}^{i}-\boldsymbol{p}_{i} \boldsymbol{d} \boldsymbol{q}^{i}+\pi^{i j}(\boldsymbol{x}) \boldsymbol{\xi}_{i} \boldsymbol{p}_{j}-\frac{1}{2} \frac{\partial \pi^{j k}}{\partial x^{i}}(\boldsymbol{x}) \boldsymbol{q}^{i} \boldsymbol{p}_{j} \boldsymbol{p}_{k}+\frac{1}{3 !} R^{i j k}(\boldsymbol{x}) \boldsymbol{p}_{i} \boldsymbol{p}_{j} \boldsymbol{p}_{k}\right) .
$$

We call (6.7) the Poisson Courant sigma model or the contravariant Courant sigma model. We take the variation of $S$,

$$
\begin{aligned}
\delta S=\int_{\mathcal{X}} \mu\left(\delta \boldsymbol{\xi}_{i} \boldsymbol{d} \boldsymbol{x}^{i}+\boldsymbol{\xi}_{i} \boldsymbol{d} \delta \boldsymbol{x}^{i}-\delta \boldsymbol{p}_{i} \boldsymbol{d} \boldsymbol{q}^{i}-\boldsymbol{p}_{i} \boldsymbol{d} \delta \boldsymbol{q}^{i}\right. \\
\left.\quad+\delta\left(\pi^{i j}(\boldsymbol{x}) \boldsymbol{\xi}_{i} \boldsymbol{p}_{j}-\frac{1}{2} \frac{\partial \pi^{j k}}{\partial x^{i}}(\boldsymbol{x}) \boldsymbol{q}^{i} \boldsymbol{p}_{j} \boldsymbol{p}_{k}+\frac{1}{3 !} R^{i j k}(\boldsymbol{x}) \boldsymbol{p}_{i} \boldsymbol{p}_{j} \boldsymbol{p}_{k}\right)\right) .
\end{aligned}
$$

The equations of motion for $\boldsymbol{\xi}$ and $\boldsymbol{q}$ are obtained by integration by parts. Since

$$
\left.\delta S\right|_{\partial \mathcal{X}}=\left.\int_{\partial \mathcal{X}} \mu_{\partial \mathcal{X}}\left(\boldsymbol{\xi}_{i} \delta \boldsymbol{x}^{i}+\boldsymbol{p}_{i} \delta \boldsymbol{q}^{i}\right)\right|_{\partial \mathcal{X}}
$$

the boundary terms, $\boldsymbol{\xi}_{i} \delta \boldsymbol{x}^{i}+\boldsymbol{p}_{i} \delta \boldsymbol{q}^{i}$, must vanish in order to derive consistent equations of motion. This equation determines the boundary conditions. It is satisfied, if for the Liouville 1-form it holds $\mathrm{ev}^{*} \vartheta=0$ on the boundary of the membrane in the target space. This means, that the Lagrangian submanifold $\mathcal{L}$ is the zero locus of $\vartheta$.

On the other hand, the boundary condition must be consistent with the classical master equation on the mapping space, $\{S, S\}_{\mathrm{BV}}=0$. Direct computation gives

$$
\begin{aligned}
\{S, S\}_{\mathrm{BV}}=\int_{\partial \mathcal{X}} \mu_{\partial \mathcal{X}}\left(\boldsymbol{\xi}_{i} \boldsymbol{d} \boldsymbol{x}^{i}-\boldsymbol{p}_{i} \boldsymbol{d} \boldsymbol{q}^{i}+\pi^{i j}(\boldsymbol{x}) \boldsymbol{\xi}_{i} \boldsymbol{p}_{j}\right. & \\
& \left.-\frac{1}{2} \frac{\partial \pi^{j k}}{\partial x^{i}}(\boldsymbol{x}) \boldsymbol{q}^{i} \boldsymbol{p}_{j} \boldsymbol{p}_{k}+\frac{1}{3 !} R^{i j k}(\boldsymbol{x}) \boldsymbol{p}_{i} \boldsymbol{p}_{j} \boldsymbol{p}_{k}\right)\left.\right|_{\partial \mathcal{X}} .
\end{aligned}
$$

We can take boundary conditions $\left.\boldsymbol{\xi}_{i}\right|_{\partial \mathcal{X}}=0$ and $\left.\boldsymbol{p}_{i}\right|_{\partial \mathcal{X}}=0$, such that (6.9) and (6.10) vanish. Therefore, in terms of the target space, the structure is $\xi_{i}=p_{i}=0$. This corresponds to the Dirac structure [12] $T^{*} M$ of the Poisson Courant algebroid $T M \oplus T^{*} M$. 
Next, we consider a more nontrivial case with boundary term, modifying the Qstructure by a canonical function $\alpha$. As an example, we take $\alpha=-\frac{1}{2} B_{i j}(x) q^{i} q^{j}$, constructed from a 2 -form $B=\frac{1}{2} B_{i j}(x) d x^{i} \wedge d x^{j}$. The Q-structure changes to

$$
\begin{aligned}
S^{\prime \prime}= & \int_{\mathcal{X}} \mu\left(\boldsymbol{\xi}_{i} \boldsymbol{d} \boldsymbol{x}^{i}-\boldsymbol{p}_{i} \boldsymbol{d} \boldsymbol{q}^{i}+\pi^{i j}(\boldsymbol{x}) \boldsymbol{\xi}_{i} \boldsymbol{p}_{j}-\frac{1}{2} \frac{\partial \pi^{j k}}{\partial x^{i}}(\boldsymbol{x}) \boldsymbol{q}^{i} \boldsymbol{p}_{j} \boldsymbol{p}_{k}+\frac{1}{3 !} R^{i j k}(\boldsymbol{x}) \boldsymbol{p}_{i} \boldsymbol{p}_{j} \boldsymbol{p}_{k}\right) \\
& +\int_{\partial \mathcal{X}} \mu_{\partial \mathcal{X}} \frac{1}{2} B_{i j}(\boldsymbol{x}) \boldsymbol{q}^{i} \boldsymbol{q}^{j},
\end{aligned}
$$

where we have used the expression $S^{\prime \prime}=e^{-\delta_{\hat{\alpha}}} S^{\prime}$ in (6.5). The boundary term deforms the boundary conditions. The variation $\delta S^{\prime \prime}$ restricted to the boundary is

$$
\left.\delta S^{\prime \prime}\right|_{\partial \mathcal{X}}=\int_{\partial \mathcal{X}} \mu_{\partial \mathcal{X}}\left[\left(\boldsymbol{\xi}_{i}+\frac{1}{2} \frac{\partial B_{j k}(\boldsymbol{x})}{\partial \boldsymbol{x}^{i}} \boldsymbol{q}^{j} \boldsymbol{q}^{k}\right) \delta \boldsymbol{x}^{i}+\left(\boldsymbol{p}_{i}-B_{i j}(\boldsymbol{x}) \boldsymbol{q}^{j}\right) \delta \boldsymbol{q}^{i}+\cdots\right] .
$$

Since these terms must vanish, consistent boundary conditions are as follows:

$$
\left.\boldsymbol{\xi}_{i}\right|_{\partial \mathcal{X}}=-\left.\frac{1}{2} \frac{\partial B_{j k}(\boldsymbol{x})}{\partial \boldsymbol{x}^{i}} \boldsymbol{q}^{j} \boldsymbol{q}^{k}\right|_{\partial \mathcal{X}},\left.\quad \boldsymbol{p}_{i}\right|_{\partial \mathcal{X}}=\left.B_{i j}(\boldsymbol{x}) \boldsymbol{q}^{j}\right|_{\partial \mathcal{X}}
$$

The master equation, $\left\{S^{\prime \prime}, S^{\prime \prime}\right\}_{\mathrm{BV}}=0$, requires another consistency condition, i.e., the integrand of $S_{1}$ is zero on the boundary,

$$
\left.\left(\pi^{i j}(\boldsymbol{x}) \boldsymbol{\xi}_{i} \boldsymbol{p}_{j}-\frac{1}{2} \frac{\partial \pi^{j k}}{\partial x^{i}}(\boldsymbol{x}) \boldsymbol{q}^{i} \boldsymbol{p}_{j} \boldsymbol{p}_{k}+\frac{1}{3 !} R^{i j k}(\boldsymbol{x}) \boldsymbol{p}_{i} \boldsymbol{p}_{j} \boldsymbol{p}_{k}\right)\right|_{\partial \mathcal{X}}=0
$$

Similarly, (6.12) and (6.13) can be converted to a condition on the target space, such that

$$
\Theta=\pi^{i j}(x) \xi_{i} p_{j}-\frac{1}{2} \frac{\partial \pi^{j k}}{\partial x^{i}}(x) q^{i} p_{j} p_{k}+\frac{1}{3 !} R^{i j k}(x) p_{i} p_{j} p_{k}=0
$$

holds on the Lagrangian submanifold $\mathcal{L}^{\prime}$, defined by

$$
\xi_{i}=-\frac{1}{2} \frac{\partial B_{j k}(x)}{\partial x^{i}} q^{j} q^{k}, \quad p_{i}=B_{i j}(x) q^{j} .
$$

Substituting (6.15) into (6.14), we obtain the following geometric structure on $\mathcal{L}^{\prime}$ :

$$
\begin{aligned}
\pi^{i j}(x) \xi_{i} p_{j} & -\frac{1}{2} \frac{\partial \pi^{j k}}{\partial x^{i}}(x) q^{i} p_{j} p_{k}+\frac{1}{3 !} R^{i j k}(x) p_{i} p_{j} p_{k} \\
= & \left(-\frac{1}{2} \pi^{l m} \frac{\partial B_{i j}}{\partial x^{l}} B_{m k}-\frac{1}{2} \frac{\partial \pi^{l m}}{\partial x^{i}} B_{j l} B_{k m}-\frac{1}{3 !} R^{l m n} B_{i l} B_{j m} B_{k n}\right) q^{i} q^{j} q^{k}=0
\end{aligned}
$$

(6.16) is equivalent to ${ }^{5}$

$$
[B, B]_{\pi}=\wedge^{3} B^{b} R .
$$

\footnotetext{
${ }^{5}$ The Koszul bracket $[-,-]_{\pi}$ is extended to the space of all differential forms as the Lie bracket satisfying the Leibniz rule. The homomorphism $B^{\mathrm{b}}: T M \rightarrow T^{*} M$ is defined by $B^{\mathrm{b}}(X)=B_{i j} X^{i}(x) \partial / \partial x^{j}$, where $X=X^{i}(x) \partial / \partial x^{i}$ is a vector field.
} 
The commutator of a 2 -form $B$ with respect to the Koszul bracket is twisted by a 3 -vector field $R$. If $B=\pi^{-1},(6.16)$ becomes

$$
H=d B=\wedge^{3} B^{b} R
$$

Next, we construct the boundary action on $T[1] \partial X$ by integrating out the superfield $\boldsymbol{\xi}_{i}$ and using the Stokes' theorem. Suppose $\pi$ is nondegenerate, i.e., $\pi^{-1}$ is symplectic. Integrating out $\boldsymbol{\xi}_{i}$ from the action (6.11), we obtain the equations of motion $\boldsymbol{p}_{i}=-\pi_{i j}^{-1} \boldsymbol{d} \boldsymbol{x}^{j}$. By substituting this equation to (6.11), the action becomes the boundary (twisted) AKSZ sigma model with WZ term in two dimensions,

$$
\begin{aligned}
S= & \int_{\partial \mathcal{X}} \mu_{\partial \mathcal{X}}\left(\pi^{-1}\right)_{i j} \boldsymbol{q}^{i} \boldsymbol{d} \boldsymbol{x}^{j}+\frac{1}{2} B_{i j}(\boldsymbol{x}) \boldsymbol{q}^{i} \boldsymbol{q}^{j} \\
& -\int_{\mathcal{X}} \mu \frac{1}{3 !} R^{i j k}(\boldsymbol{x})\left(\pi^{-1}\right)_{i l}\left(\pi^{-1}\right)_{j m}\left(\pi^{-1}\right)_{k n} \boldsymbol{d} \boldsymbol{x}^{l} \boldsymbol{d} \boldsymbol{x}^{m} \boldsymbol{d} \boldsymbol{x}^{n} .
\end{aligned}
$$

This is the Poisson sigma model [45-47] deformed by a WZ term [48].

The action without ghosts can be obtained as follows. We expand the superfields in components as

$$
\mathbf{\Phi}(\sigma, \theta)=\Phi^{(0)}(\sigma)+\Phi_{\mu}^{(1)}(\sigma) \theta^{\mu}+\frac{1}{2} \Phi_{\mu \nu}^{(2)}(\sigma) \theta^{\mu} \theta^{\nu}
$$

If we integrate over $\theta^{\mu}$ and drop ghost fields with nonzero degrees, we get the physical action:

$$
\begin{aligned}
S= & -\int_{\partial X}\left(\left(\pi^{-1}\right)_{i j} q^{i} \wedge d x^{j}-\frac{1}{2} B_{i j}(x) q^{i} \wedge q^{j}\right) \\
& -\int_{X} \frac{1}{3 !} R^{i j k}(x)\left(\pi^{-1}\right)_{i l}\left(\pi^{-1}\right)_{j m}\left(\pi^{-1}\right)_{k n} d x^{l} \wedge d x^{m} \wedge d x^{n},
\end{aligned}
$$

where $x^{i}=x^{(0) i}$ and $q^{i}=d \sigma^{\mu} q_{\mu}^{(1) i}$. If we add the kinetic term, we obtain a string sigma model action with R-flux,

$$
\begin{aligned}
S= & \frac{1}{2} \int_{\partial X} G_{i j}(x) d x^{i} \wedge * d x^{j}-\int_{\partial X}\left(\left(\pi^{-1}\right)_{i j} q^{i} \wedge d x^{j}-\frac{1}{2} B_{i j}(x) q^{i} \wedge q^{j}\right) \\
& -\int_{X} \frac{1}{3 !} R^{i j k}(x)\left(\pi^{-1}\right)_{i l}\left(\pi^{-1}\right)_{j m}\left(\pi^{-1}\right)_{k n} d x^{l} \wedge d x^{m} \wedge d x^{n} .
\end{aligned}
$$

\subsection{Duality of Courant sigma models}

We have discussed duality transformations of the standard and the Poisson Courant algebroids in section 4 . In this subsection, we derive the same result from the analysis of the corresponding sigma models.

We perform the duality transformation on the level of sigma models. The AKSZ construction on a three-dimensional manifold $X$ with boundary gives rise to two Courant sigma models, one with the Poisson Courant algebroid structure with $R$-flux, constructed in the previous subsection, and one with the standard Courant algebroid structure with $H$-flux. 
The BV action of the Poisson Courant sigma model is (6.7). On the other hand, from the AKSZ construction, the BV action of the standard Courant sigma model is

$$
S=\int_{\mathcal{X}} \mu\left(\boldsymbol{\xi}_{i} \boldsymbol{d} \boldsymbol{x}^{i}-\boldsymbol{p}_{i} \boldsymbol{d} \boldsymbol{q}^{i}+\boldsymbol{\xi}_{i} \boldsymbol{q}^{i}+\frac{1}{3 !} H_{i j k}(\boldsymbol{x}) \boldsymbol{q}^{i} \boldsymbol{q}^{j} \boldsymbol{q}^{k}\right) .
$$

We consider the following twisting of the standard Courant sigma model by applying the twist (4.3) as

$$
S^{\prime}=\int_{\mathcal{X}} \mu\left(\boldsymbol{\xi}_{i} \boldsymbol{d} \boldsymbol{x}^{i}-\boldsymbol{q}^{i} \boldsymbol{d} \boldsymbol{p}_{i}\right)+e^{\delta_{\alpha_{p}}} e^{-\delta_{\alpha_{q}}} e^{\delta_{\alpha_{p}}} \int_{\mathcal{X}} \mu\left(\boldsymbol{\xi}_{i} \boldsymbol{q}^{i}+\frac{1}{3 !} H_{i j k}(\boldsymbol{x}) \boldsymbol{q}^{i} \boldsymbol{q}^{j} \boldsymbol{q}^{k}\right) .
$$

This is equivalent to

$$
S^{\prime \prime}=e^{-\delta_{\alpha_{p}}} e^{\delta_{\alpha_{q}}} e^{-\delta_{\alpha_{p}}} \int_{\mathcal{X}} \mu\left(\boldsymbol{\xi}_{i} \boldsymbol{d} \boldsymbol{x}^{i}-\boldsymbol{q}^{i} \boldsymbol{d} \boldsymbol{p}_{i}\right)+\int_{\mathcal{X}} \mu\left(\boldsymbol{\xi}_{i} \boldsymbol{q}^{i}+\frac{1}{3 !} H_{i j k}(\boldsymbol{x}) \boldsymbol{q}^{i} \boldsymbol{q}^{j} \boldsymbol{q}^{k}\right),
$$

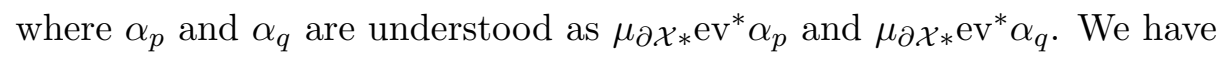

$$
\begin{aligned}
& e^{-\delta_{\alpha_{p}}} e^{\delta_{\alpha_{q}}} e^{-\delta_{\alpha_{p}}} S_{0}=S_{0}+\int_{\partial \mathcal{X}} \mu_{\partial \mathcal{X}}\left(\frac{1}{2} \pi^{i j}(\boldsymbol{x}) \boldsymbol{p}_{i} \boldsymbol{p}_{j}+\boldsymbol{p}_{i} \boldsymbol{q}^{i}\right) \\
& -\int_{\partial \mathcal{X}} \mu_{\partial \mathcal{X}}\left(-\frac{1}{2} \pi^{i j}(\boldsymbol{x}) \boldsymbol{p}_{i} \boldsymbol{p}_{j}+\pi^{i j}(\boldsymbol{x}) \boldsymbol{p}_{i} \boldsymbol{p}_{j}\right) \\
& =S_{0}+\int_{\partial \mathcal{X}} \mu_{\partial \mathcal{X}}\left(\boldsymbol{p}_{i} \boldsymbol{q}^{i}\right) \text {. }
\end{aligned}
$$

Therefore, by this twist, the action becomes the Courant sigma model with boundary term,

$$
S^{\prime \prime}=\int_{\mathcal{X}} \mu\left(\boldsymbol{\xi}_{i} \boldsymbol{d} \boldsymbol{x}^{i}-\boldsymbol{q}^{i} \boldsymbol{d} \boldsymbol{p}_{i}+\boldsymbol{\xi}_{i} \boldsymbol{q}^{i}+\frac{1}{3 !} H_{i j k}(\boldsymbol{x}) \boldsymbol{q}^{i} \boldsymbol{q}^{j} \boldsymbol{q}^{k}\right)+\int_{\partial \mathcal{X}} \mu_{\partial \mathcal{X}}\left(\boldsymbol{p}_{i} \boldsymbol{q}^{i}\right) .
$$

From (4.4)-(4.7), redefining the superfields as

$$
\begin{aligned}
\boldsymbol{x}^{i} & =\boldsymbol{x}^{\prime i} \\
\boldsymbol{q}^{i} & =\pi^{i j}(\boldsymbol{x}) \boldsymbol{p}_{j}^{\prime}, \\
\boldsymbol{p}_{i} & =-\pi_{i j}^{-1}(\boldsymbol{x}) \boldsymbol{q}^{\prime j}, \\
\boldsymbol{\xi}_{i} & =\boldsymbol{\xi}_{i}^{\prime}+\frac{\partial \pi^{j k}}{\partial x^{i}} \pi_{k l}^{-1}(\boldsymbol{x}) \boldsymbol{p}^{\prime}{ }_{j} \boldsymbol{q}^{\prime},
\end{aligned}
$$

we can simplify the total action as

$$
\begin{aligned}
S^{\prime \prime}=\int_{\mathcal{X}} \mu\left(\boldsymbol{\xi}_{i}^{\prime} \boldsymbol{d} \boldsymbol{x}^{i}-\boldsymbol{p}_{i}^{\prime} \boldsymbol{d}^{\boldsymbol{\prime}^{i}}\right. & \\
& \\
& \left.+\pi^{i j}(\boldsymbol{x}) \boldsymbol{\xi}_{i}^{\prime} \boldsymbol{p}^{\prime}{ }_{j}-\frac{1}{2} \frac{\partial \pi^{j k}}{\partial x^{i}}(\boldsymbol{x}){\boldsymbol{\boldsymbol { q } ^ { \prime }}}^{i} \boldsymbol{p}^{\prime}{ }_{j} \boldsymbol{p}^{\prime}{ }_{k}+\frac{1}{3 !} R^{i j k}(\boldsymbol{x}) \boldsymbol{p}_{i}^{\prime} \boldsymbol{p}^{\prime}{ }_{j} \boldsymbol{p}^{\prime}{ }_{k}\right) .
\end{aligned}
$$

The resulting action is the same as the BV action of the Poisson (contravariant) Courant sigma model (6.7), and we obtain the relation between $H$ and $R, R=\wedge^{3} \pi^{\sharp} H$, again. Therefore, in the theory of the topological membrane, the duality transformation between $H$-flux and $R$-flux is a change of boundary conditions. 


\section{Current algebras}

In this section, we consider a current algebra à la Alekseev and Strobl [27, 32, 33, 51] corresponding to the Poisson Courant algebroid in two-dimensional spacetime.

\subsection{Poisson brackets with fluxes from target QP-structures}

In this subsection, we briefly review the Hamiltonian method to construct a Poisson bracket of canonical variables with fluxes from general target QP-structures [27].

Let $(\mathcal{M}, \omega)$ be a P-manifold of degree $n-1$. We take a worldvolume $X=$ $\Sigma \times \boldsymbol{R}$ in $n$ dimensions and a space supermanifold $\mathcal{X}=T[1] \Sigma$, since we consider the Hamiltonian formalism.

The simplest method to determine a Poisson bracket is to construct it from $\boldsymbol{\omega}=$ $\mu_{*} \mathrm{ev}^{*} \omega$. Since $\Sigma$ is in $n-1$ dimensions and $\omega$ is of degree $n-1$, the graded symplectic structure $\boldsymbol{\omega}$ is of degree zero due to the integration $\mu_{*}$. Then, we obtain a graded Poisson bracket of degree zero, that is, an ordinary Poisson bracket $\{-,-\}_{\mathrm{PB}}$. However, the Poisson brackets obtained in this way cannot include fluxes, since the target space geometric datum $\Theta$ is not used. In [32], the symplectic form was deformed by $b$-transformation to include H-flux.

Here, we use another method to incorporate the geometric datum $\Theta$. First we consider a QP-manifold of degree $n,(\mathcal{M}, \omega, \Theta)$, as an extended target space. A Lagrangian submanifold $\mathcal{L}$ with respect to $\omega$ is regarded as the target space of physical canonical quantities. $\omega$ defines a graded Poisson bracket, $\{-,-\}$, of degree $-n$.

Then, we consider the derived bracket $\{\{-, \Theta\},-\}$, which is of degree $-n+1$, and use the following fact.

Theorem 7.1. Let $(\mathcal{M}, \omega, Q)$ be a QP-manifold, $\mathcal{L}$ a Lagrangian submanifold with respect to $\omega$, and pr: $\mathcal{M} \rightarrow \mathcal{L}$ the natural projection. If the derived bracket is restricted to $\mathcal{L}$, then it gives the graded Poisson bracket $\left.\{f, g\}_{\mathcal{L}} \equiv\left\{\left\{p r^{*} f, \Theta\right\}, p r^{*} g\right\}\right|_{\mathcal{L}}$ for functions $f$ and $g$ on $\mathcal{L}$.

We can easily prove that $\{f, g\}_{\mathcal{L}}$ is antisymmetric and satisfies both the Leibniz rule and the Jacobi identity using $\left\{p r^{*} f, p r^{*} g\right\}=0$.

In order to define a Poisson bracket on the mapping space, we transgress the derived bracket to the mapping space $\operatorname{Map}(\mathcal{X}, \mathcal{M})$. Then, the derived bracket $\left\{\left\{-, S_{1}\right\},-\right\}$ of degree zero, with $S_{1}=\mu_{*} \mathrm{ev}^{*} \Theta$, defines the Poisson bracket on the mapping space,

$$
\{F, G\}_{\mathrm{PB}}=\left.\left\{\left\{F, S_{1}\right\}, G\right\}\right|_{\widehat{\mathcal{L}}}
$$

where $F, G$ are functions on the mapping space. Precisely speaking, for a function $f \in C^{\infty}(\mathcal{L})$, we introduce test functions $\epsilon$ on $\mathcal{X}$ of degree $n-1-|f|$. A Lagrangian submanifold of $\operatorname{Map}(\mathcal{X}, \mathcal{M})$ is denoted by $\widehat{\mathcal{L}}$, and the projection map is $\widehat{p r}: \operatorname{Map}(\mathcal{X}, \mathcal{M}) \rightarrow \widehat{\mathcal{L}}$. We can prove that the restriction of the derived bracket to a Lagrangian submanifold $\widehat{\mathcal{L}}$, $\widehat{p r}_{*}\left\{\left\{-, S_{1}\right\},-\right\}=\left.\left\{\left\{-, S_{1}\right\},-\right\}\right|_{\widehat{\mathcal{L}}}$, becomes an ordinary Poisson bracket $\{-,-\}_{\mathrm{PB}}$,

$$
\left\{\mu_{*} \epsilon_{1} \mathrm{ev}^{*} p r^{*} f, \mu_{*} \epsilon_{2} \mathrm{ev}^{*} p r^{*} g\right\}_{\mathrm{PB}}=\widehat{p r}_{*}\left\{\left\{\mu_{*} \epsilon_{1} \mathrm{ev}^{*} p r^{*} f, S_{1}\right\}, \mu_{*} \epsilon_{2} \mathrm{ev}^{*} p r^{*} g\right\} .
$$


The Poisson bracket depends not only on $\Theta$, but also on the choice of the Lagrangian submanifold $\widehat{\mathcal{L}}$.

Note that we can use the following formula to connect the target space computations to the superfield computations,

$$
\left\{\left\{\mu_{*} \epsilon_{1} \mathrm{ev}^{*} p r^{*} f, S_{1}\right\}, \mu_{*} \epsilon_{2} \mathrm{ev}^{*} p r^{*} g\right\}=\mu_{*} \epsilon_{1} \epsilon_{2} \mathrm{ev}^{*}\left\{\left\{p r^{*} f, \Theta\right\}, p r^{*} g\right\}
$$

where $f, g \in C^{\infty}(\mathcal{L})$.

Simple candidates for $\widehat{\mathcal{L}}$ are canonical Lagrangian submanifolds, which we denote by $\widehat{\mathcal{L}}_{0}$. For instance, in the case of the Courant algebroids, two simple Lagrangian submanifolds in $\operatorname{Map}\left(\mathcal{X}, T^{*}[2] T^{*}[1] M\right)$ are $\operatorname{Map}\left(\mathcal{X}, T^{*}[1] M\right)=\left\{\left(\boldsymbol{x}^{i}, \boldsymbol{p}_{i}, \boldsymbol{q}^{i}, \boldsymbol{\xi}_{i}\right) \mid \boldsymbol{\xi}_{i}=\boldsymbol{q}^{i}=0\right\}$ or $\operatorname{Map}(\mathcal{X}, T[1] M)=\left\{\left(\boldsymbol{x}^{i}, \boldsymbol{p}_{i}, \boldsymbol{q}^{i}, \boldsymbol{\xi}_{i}\right) \mid \boldsymbol{\xi}_{i}=\boldsymbol{p}_{i}=0\right\}$. Generally, we cannot obtain the twisted Poisson bracket with fluxes by simple restriction to these canonical Lagrangian submanifolds. We do a special twisting of functions on the mapping space, before restricting the space to a canonical Lagrangian submanifold, where the twisting does not depend on fluxes. This procedure derives a Poisson bracket with flux.

The space of functions on a canonical Lagrangian submanifold is constructed as $C^{\infty}\left(\widehat{\mathcal{L}}_{0}\right) \equiv\left\{\widehat{p r}_{*} \mu_{*} \epsilon \mathrm{ev}^{*} p r^{*} f \mid f \in C^{\infty}\left(\mathcal{L}_{0}\right)\right\}$. We twist the functions to be $e^{\delta_{\alpha}} \mu_{*} \epsilon \mathrm{ev}^{*} p r^{*} f=e^{\delta_{\alpha}} \int_{\mathcal{X}} \mu \epsilon(\sigma, \theta) f(\boldsymbol{p}(\sigma, \theta))$. The twisted derived bracket $\left\{\left\{e^{\delta_{\alpha}} \mu_{*} \epsilon \mathrm{ev}^{*} p r^{*} f, S_{1}\right\}, e^{\delta_{\alpha}} \mu_{*} \epsilon \mathrm{ev}^{*} p r^{*} g\right\}$ restricted to $\widehat{\mathcal{L}}_{0}$ gives rise to a Poisson bracket with fluxes, if we choose $\alpha$ properly. The formula is

$$
\left\{e^{\delta_{\alpha}} \mu_{*} \epsilon_{1} \mathrm{ev}^{*} p r^{*} f, e^{\delta_{\alpha}} \mu_{*} \epsilon_{2} \mathrm{ev}^{*} p r^{*} g\right\}_{\mathrm{PB}} \equiv \widehat{p r}_{*}\left\{\left\{e^{\delta_{\alpha}} \mu_{*} \epsilon_{1} \mathrm{ev}^{*} p r^{*} f, S_{1}\right\}, e^{\delta_{\alpha}} \mu_{*} \epsilon_{2} \mathrm{ev}^{*} p r^{*} g\right\} .
$$

Assume that a nontwisted Poisson bracket $\{-,-\}_{\mathrm{PB}}$ on the canonical Lagrangian submanifold is nondegenerate, that is, symplectic. ${ }^{6}$ Then, there exists a graded symplectic form $\boldsymbol{\omega}_{\widehat{\mathcal{L}}_{0}}=\mu_{*} \mathrm{ev}^{*} \omega_{\mathcal{L}_{0}}$ on $\widehat{\mathcal{L}}_{0}$ corresponding to this Poisson bracket. We choose $\alpha_{0}=$ ${ }^{\iota}{ }_{\hat{D}} \mu_{*} \mathrm{ev}^{*} \vartheta_{\mathcal{L}_{0}}$ as the twisting function, where $\vartheta_{\mathcal{L}_{0}}$ is the Liouville 1-form, such that $\omega_{\mathcal{L}_{0}}=$ $-\delta \vartheta_{\mathcal{L}_{0}}$. Note that $\alpha_{0}$ does not depend on the fluxes.

Here, we have twisted the space of functions on the mapping space and restricted it to the canonical Lagrangian submanifold. We could also obtain the same Poisson bracket by twisting the canonical Lagrangian submanifold with a canonical transformation $e^{-\delta_{\alpha}}$ and restricting the derived bracket to the twisted Lagrangian submanifold.

We demonstrate the procedure in the $H$-flux case.

Example 7.1 (Poisson brackets twisted by H-flux). We construct the Poisson bracket of the standard Courant algebroid with $H$-flux on the cotangent space of the loop space $\operatorname{Map}\left(S^{1}, T^{*} M\right)$. First we determine the Poisson brackets of the canonical quantities.

Let us consider the QP-manifold of the standard Courant algebroid, $\mathcal{M}=T^{*}[2] T^{*}[1] M$, in section 2. Take local Darboux coordinates $\left(x^{i}, p_{i}, q^{i}, \xi_{i}\right)$ of degree $(0,1,1,2)$. The canonical graded symplectic structure is expressed by $\omega=\delta x^{i} \wedge \delta \xi_{i}+\delta p_{i} \wedge \delta q^{i}$. We take a canonical Lagrangian submanifold with respect to $\omega, \mathcal{L}_{0}=\left\{\left(x^{i}, p_{i}\right) \mid \xi_{i}=q^{i}=0\right\}$.

\footnotetext{
${ }^{6}$ This condition is satisfied, if $\mathcal{M}$ is a double graded cotangent bundle of a graded manifold $\mathcal{N}$, for example, $\mathcal{M}=T^{*}[n] T^{*}[n-1] \mathcal{N}$. All QP-manifolds in this paper are double cotangent bundles.
} 
Since the Q-structure function of the standard Courant algebroid is $\Theta=\xi_{i} q^{i}+$ $\frac{1}{3 !} H_{i j k}(x) q^{i} q^{j} q^{k}$, the derived brackets for the canonical quantities on $\mathcal{L}_{0}$ are

$$
\begin{aligned}
& \left\{\left\{x^{i}, \Theta\right\}, x^{j}\right\}=0 \\
& \left\{\left\{x^{i}, \Theta\right\}, p_{j}\right\}=\delta^{i}{ }_{j}, \\
& \left\{\left\{p_{i}, \Theta\right\}, p_{j}\right\}=-H_{i j k}(x) q^{k} .
\end{aligned}
$$

Next, we consider the mapping space. Take the supermanifold $\mathcal{X}=T[1] S^{1}$ with local coordinates $(\sigma, \theta)$. Local coordinates on $\operatorname{Map}\left(T[1] S^{1}, T^{*}[2] T[1] M\right)$ are superfields $\boldsymbol{x}^{i}(\sigma, \theta): T[1] S^{1} \rightarrow M$ and $\boldsymbol{q}^{i}(\sigma, \theta) \in \Gamma\left(T^{*}[1] S^{1} \otimes \boldsymbol{x}^{*}\left(T_{x}[1] M\right)\right)$ of degree $(0,1)$ and canonical conjugates $\boldsymbol{\xi}_{i}(\sigma, \theta)$ and $\boldsymbol{p}_{i}(\sigma, \theta)$ of degree $(2,1), \boldsymbol{\xi}_{i}(\sigma, \theta) \in \Gamma\left(T^{*}[1] S^{1} \otimes \boldsymbol{x}^{*}\left(T_{x}^{*}[2] M\right)\right)$ and $\boldsymbol{p}_{i}(\sigma, \theta) \in \Gamma\left(T^{*}[1] S^{1} \otimes \boldsymbol{x}^{*}\left(T_{q}^{*}[2] T_{x}[1] M\right)\right)$.

The transgression of (7.5)-(7.7) induces the derived bracket on superfields. The concrete expression is

$$
\begin{aligned}
& \left\{\left\{\boldsymbol{x}^{i}(\sigma, \theta), S_{1}\right\}, \boldsymbol{x}^{j}\left(\sigma^{\prime}, \theta^{\prime}\right)\right\}=0, \\
& \left\{\left\{\boldsymbol{x}^{i}(\sigma, \theta), S_{1}\right\}, \boldsymbol{p}_{j}\left(\sigma^{\prime}, \theta^{\prime}\right)\right\}=-\delta^{i}{ }_{j} \delta\left(\sigma-\sigma^{\prime}\right) \delta\left(\theta-\theta^{\prime}\right), \\
& \left\{\left\{\boldsymbol{p}_{i}(\sigma, \theta), S_{1}\right\}, \boldsymbol{p}_{j}\left(\sigma^{\prime}, \theta^{\prime}\right)\right\}=H_{i j k}(\boldsymbol{x}) \boldsymbol{q}^{k}(\sigma, \theta) \delta\left(\sigma-\sigma^{\prime}\right) \delta\left(\theta-\theta^{\prime}\right) .
\end{aligned}
$$

The Liouville 1-form on the Lagrangian submanifold is $\alpha_{0}=\iota^{\iota} \mu_{*} \mathrm{ev}^{*} \vartheta_{\mathcal{L}}=-\int \mu \boldsymbol{p}_{i} \boldsymbol{d} \boldsymbol{x}^{i}$. Twisting by the Liouville 1 -form $\alpha_{0}$ gives rise to the transformation $\boldsymbol{q}^{k} \rightarrow \boldsymbol{q}^{k}-\boldsymbol{d} \boldsymbol{x}^{k}$. If we reduce to the canonical Lagrangian submanifold $\widehat{\mathcal{L}}_{0}$ defined by $\boldsymbol{\xi}_{i}=\boldsymbol{q}^{i}=0$, we obtain

$$
\begin{aligned}
& \left\{\boldsymbol{x}^{i}(\sigma, \theta), \boldsymbol{x}^{j}\left(\sigma^{\prime}, \theta^{\prime}\right)\right\}_{\mathrm{PB}}=0 \\
& \left\{\boldsymbol{x}^{i}(\sigma, \theta), \boldsymbol{p}_{j}\left(\sigma^{\prime}, \theta^{\prime}\right)\right\}_{\mathrm{PB}}=-\delta^{i}{ }_{j} \delta\left(\sigma-\sigma^{\prime}\right) \delta\left(\theta-\theta^{\prime}\right), \\
& \left\{\boldsymbol{p}_{i}(\sigma, \theta), \boldsymbol{p}_{j}\left(\sigma^{\prime}, \theta^{\prime}\right)\right\}_{\mathrm{PB}}=-H_{i j k}(\boldsymbol{x}(\sigma, \theta)) \boldsymbol{d} \boldsymbol{x}^{k}(\sigma, \theta) \delta\left(\sigma-\sigma^{\prime}\right) \delta\left(\theta-\theta^{\prime}\right) .
\end{aligned}
$$

We expand the superfields by the local coordinate $\theta$ on $T[1] S^{1}$,

$$
\boldsymbol{\Phi}(\sigma, \theta)=\Phi^{(0)}(\sigma)+\Phi^{(1)}(\sigma) \theta .
$$

The degree zero component in the expansion is the physical field (and degree nonzero components are ghost fields). In this example, physical fields are $x^{i}(\sigma)=x^{(0) i}(\sigma)$ and $p_{i}(\sigma)=p_{i}^{(1)}(\sigma)$. The Poisson brackets of the physical canonical quantities are degree zero components of (7.11)-(7.13):

$$
\begin{aligned}
& \left\{x^{i}(\sigma), x^{j}\left(\sigma^{\prime}\right)\right\}_{\mathrm{PB}}=0 \\
& \left\{x^{i}(\sigma), p_{j}\left(\sigma^{\prime}\right)\right\}_{\mathrm{PB}}=\delta^{i}{ }_{j} \delta\left(\sigma-\sigma^{\prime}\right) \\
& \left\{p_{i}(\sigma), p_{j}\left(\sigma^{\prime}\right)\right\}_{\mathrm{PB}}=-H_{i j k}(x) \partial_{\sigma} x^{k}(\sigma) \delta\left(\sigma-\sigma^{\prime}\right) .
\end{aligned}
$$

These are the Poisson brackets of the canonical quantities with H-flux in [32]. The symplectic form of Alekseev-Strobl type, which induces (7.15)-(7.17), is

$$
\omega=\int_{S^{1}} d \sigma \delta x^{i} \wedge \delta p_{i}+\frac{1}{2} \int_{S^{1}} d \sigma H_{i j k}(x) \partial_{\sigma} x^{i} \delta x^{j} \wedge \delta x^{k} .
$$




\subsection{Current algebras from target QP-structures}

A current algebra is a Poisson algebra on the mapping space. Consider a subspace of $C^{\infty}(\mathcal{M})$, which is closed under the derived bracket $\{\{-, \Theta\},-\}$. This subspace becomes a Poisson algebra on the mapping space after transgression. The finite degree subspace, $\sum_{i=0}^{n-1} C_{i}(\mathcal{M})=\left\{f \in C^{\infty}(\mathcal{M})|| f \mid \leq n-1\right\}$, is closed under the derived bracket. If $C_{n-1}(\mathcal{M})$ is transgressed to $\operatorname{Map}(\mathcal{X}, \mathcal{M})$, twisted by $\alpha_{0}$ and restricted to the Lagrangian submanifold $\widehat{\mathcal{L}}_{0}$, we obtain a current algebra with fluxes.

For the $H$-flux case, we demonstrate the construction in the following example.

Example 7.2 (Alekseev-Strobl current algebras). Based on the result in example 7.1, we take $C_{0} \oplus C_{1}=\left\{f \in C^{\infty}\left(T^{*}[2] T^{*}[1] M\right)|| f \mid \leq 1\right\}$ as space of functions. Elements of $C_{0} \oplus C_{1}$ are functions of degree zero, $j_{0(f)}=f(x)$ and functions of degree one, $j_{1(X+\alpha)}=$ $\alpha_{i}(x) q^{i}+X^{i}(x) p_{i} \cdot j_{1(X+\alpha)}$ is equivalent to a section of $T M \oplus T^{*} M$, since it corresponds to $\alpha_{i}(x) d x^{i}+X^{i}(x) \frac{\partial}{\partial x^{i}}$. Let $j_{(0)(g)}=g(x)$ and $j_{(1)(Y+\beta)}=\beta_{i}(x) q^{i}+Y^{i}(x) p_{i}$. The derived brackets of functions are easily computed,

$$
\begin{aligned}
\left\{\left\{j_{0(f)}, \Theta\right\}, j_{0(g)}\right\} & =0 \\
\left\{\left\{j_{1(X+\alpha)}, \Theta\right\}, j_{0(g)}\right\} & =-X^{i} \frac{\partial j_{0(g)}}{\partial x^{i}}=-\rho(X+\alpha) j_{0(g)}, \\
\left\{\left\{j_{1(X+\alpha)}, \Theta\right\}, j_{1(Y+\beta)}\right\} & =-j_{1\left([X+\alpha, Y+\beta]_{H}\right)},
\end{aligned}
$$

where $[X+\alpha, Y+\beta]_{H}$ is the Dorfman bracket on the standard Courant algebroid with flux $H$,

$$
[X+\alpha, Y+\beta]_{H}=[X, Y]+L_{X} \beta-\iota_{Y} d \alpha+\iota_{X} \iota_{Y} H
$$

for $X, Y \in \Gamma(T M), \alpha, \beta \in \Gamma\left(T^{*} M\right)$.

Currents are identified with twisted functions on the Lagrangian submanifold of the mapping space. In order to construct currents, we apply the transgression map to $j_{0}$ and $j_{1}$. Then, we twist them by $\alpha_{0}$ and finally restrict the resulting functions to the Lagrangian submanifold defined by $\boldsymbol{\xi}_{i}=\boldsymbol{q}^{i}=0$. The corresponding currents are

$$
\begin{aligned}
\boldsymbol{J}_{(0)(f)}\left(\epsilon_{(1)}\right) & =\tilde{p r}{ }_{*} e^{\delta_{\alpha_{0}}} \mu_{*} \epsilon_{(1)} \mathrm{ev}^{*} j_{(0)(f)}=\int_{T[1] S^{1}} \mu \epsilon_{(1)} f(\boldsymbol{x}), \\
\boldsymbol{J}_{(1)(X+\alpha)}\left(\epsilon_{(0)}\right) & =\tilde{p r} r_{*} e^{\delta_{\alpha_{0}}} \mu_{*} \epsilon_{(0)} \mathrm{ev}^{*} j_{(1)(u, \alpha)}=\int_{T[1] S^{1}} \mu \epsilon_{(0)}\left(-\alpha_{i}(\boldsymbol{x}) \boldsymbol{d} \boldsymbol{x}^{i}+X^{i}(\boldsymbol{x}) \boldsymbol{p}_{i}\right),
\end{aligned}
$$

where $\epsilon_{(i)}=\epsilon_{(i)}(\sigma, \theta)$ is a test function of degree $i$ on the super circle $\mathcal{X}=T[1] S^{1}$. The integrands of degree zero components of $\boldsymbol{J}_{0}$ and $\boldsymbol{J}_{1}$ are

$$
J_{(0)(f)}(\sigma)=f(x(\sigma)), \quad J_{(1)(X+\alpha)}(\sigma)=\alpha_{i}(x) \partial_{\sigma} x^{i}(\sigma)+X^{i}(x) p_{i}(\sigma),
$$

which are the correct AS currents. 
We compute the Poisson algebra of these supergeometric currents from the Poisson brackets of canonical quantities $\left(\boldsymbol{x}^{i}, \boldsymbol{p}_{i}\right)$ obtained in example 7.1:

$$
\begin{aligned}
\left\{\boldsymbol{J}_{0(f)}(\epsilon), \boldsymbol{J}_{0(g)}\left(\epsilon^{\prime}\right)\right\}_{\mathrm{PB}}= & 0 \\
\left\{\boldsymbol{J}_{1(X+\alpha)}(\epsilon), \boldsymbol{J}_{0(g)}\left(\epsilon^{\prime}\right)\right\}_{\mathrm{PB}}= & \rho(X+\alpha) \boldsymbol{J}_{0(g)}\left(\epsilon \epsilon^{\prime}\right), \\
\left\{\boldsymbol{J}_{1(X+\alpha)}(\epsilon), \boldsymbol{J}_{1(Y+\beta)}\left(\epsilon^{\prime}\right)\right\}_{\mathrm{PB}}= & \boldsymbol{J}_{1\left([X+\alpha, Y+\beta]_{H}\right)}\left(\epsilon \epsilon^{\prime}\right) \\
& +\int_{T[1] S^{1}} \mu \boldsymbol{d} \epsilon_{(0)} \epsilon_{(0)}^{\prime}\langle X+\alpha, Y+\beta\rangle(\boldsymbol{x}),
\end{aligned}
$$

where $\boldsymbol{J}_{0(g)}^{\prime}=\int_{T[1] S^{1}} \mu \epsilon_{(1)} g(\boldsymbol{x}), \boldsymbol{J}_{1(Y+\beta)}^{\prime}=\int_{T[1] S^{1}} \mu \epsilon_{(0)}\left(-\beta_{i}(\boldsymbol{x}) \boldsymbol{d} \boldsymbol{x}^{i}+Y^{i}(\boldsymbol{x}) \boldsymbol{p}_{i}\right)$. The AS current algebra is given by the physical components of the supergeometric currents, i.e., degree zero components, in (7.23)-(7.25):

$$
\begin{aligned}
\left\{J_{0(f)}(\sigma), J_{0(g)}\left(\sigma^{\prime}\right)\right\}_{\mathrm{PB}}= & 0 \\
\left\{J_{1(X+\alpha)}(\sigma), J_{0(g)}\left(\sigma^{\prime}\right)\right\}_{\mathrm{PB}}= & -\rho(X+\alpha) J_{0(g)}(x(\sigma)) \delta\left(\sigma-\sigma^{\prime}\right), \\
\left\{J_{1(X+\alpha)}(\sigma), J_{1(Y+\beta)}\left(\sigma^{\prime}\right)\right\}_{\mathrm{PB}}= & -J_{1\left([X+\alpha, Y+\beta]_{H}\right)}(\sigma) \delta\left(\sigma-\sigma^{\prime}\right) \\
& +\langle(X+\alpha),(Y+\beta)\rangle\left(\sigma^{\prime}\right) \partial_{\sigma} \delta\left(\sigma-\sigma^{\prime}\right) .
\end{aligned}
$$

This coincides with the generalized current algebra described in [32].

\subsection{Poisson bracket twisted by $R$-flux}

By the same method as in the previous section, we derive a current algebra with $R$-flux from the supergeometric data of the Poisson Courant algebroid.

Let us consider a two-dimensional worldsheet $\Sigma=S^{1} \times \boldsymbol{R}$ and take the tangent bundle $T L M=\operatorname{Map}\left(S^{1}, T M\right)$ of the loop space $L M=\operatorname{Map}\left(S^{1}, M\right)$ as phase space, dual to the cotangent bundle $T^{*} L M=\operatorname{Map}\left(S^{1}, T^{*} M\right)$ in the standard Courant algebroid case. Let $x^{i}(\sigma)$ be local coordinates on the base and $q^{i}(\sigma)$ local coordinates on the fiber, where $\sigma$ parametrizes $S^{1}$.

We would like to construct Poisson brackets between $x^{i}(\sigma)$ and $q^{i}(\sigma)$. For that, we use the QP-manifold of the Poisson Courant algebroid, which is $\mathcal{M}=T^{*}[2] T^{*}[1] M$ with Q-structure $\Theta=\pi^{i j}(x) \xi_{i} p_{j}-\frac{1}{2} \frac{\partial \pi^{j k}}{\partial x^{i}}(x) q^{i} p_{j} p_{k}+\frac{1}{3 !} R^{i j k}(x) p_{i} p_{j} p_{k}$. We take a Lagrangian submanifold $\mathcal{L}_{0}=T[1] M$ of $\mathcal{M}$ parametrized by local coordinates $\left(x^{i}, q^{i}\right)$. The derived bracket on $T[1] M$ is

$$
\begin{aligned}
& \left\{\left\{x^{i}, \Theta\right\}, x^{j}\right\}=0, \\
& \left\{\left\{x^{i}, \Theta\right\}, q^{j}\right\}=\pi^{i j}(x), \\
& \left\{\left\{q^{i}, \Theta\right\}, q^{j}\right\}=-R^{i j k}(x) p_{k}+\frac{\partial \pi^{i j}}{\partial x^{k}}(x) q^{k} .
\end{aligned}
$$

The transgression of (7.29)-(7.31) gives the derived brackets on the superfields. If we restrict them without twisting to the canonical Lagrangian submanifold parametrized by 
$\boldsymbol{\xi}_{i}=\boldsymbol{p}_{i}=0$, we obtain the Poisson bracket without $R$-flux,

$$
\begin{aligned}
& \left\{\boldsymbol{x}^{i}(\sigma, \theta), \boldsymbol{x}^{j}\left(\sigma^{\prime}, \theta^{\prime}\right)\right\}_{\mathrm{PB}}=0, \\
& \left\{\boldsymbol{x}^{i}(\sigma, \theta), \boldsymbol{q}^{j}\left(\sigma^{\prime}, \theta^{\prime}\right)\right\}_{\mathrm{PB}}=-\pi^{i j}(\boldsymbol{x}(\sigma, \theta)) \delta\left(\sigma-\sigma^{\prime}\right) \delta\left(\theta-\theta^{\prime}\right), \\
& \left\{\boldsymbol{q}^{i}(\sigma, \theta), \boldsymbol{q}^{j}\left(\sigma^{\prime}, \theta^{\prime}\right)\right\}_{\mathrm{PB}}=-\frac{\partial \pi^{i j}}{\partial \boldsymbol{x}^{k}}(\boldsymbol{x}(\sigma, \theta)) \boldsymbol{q}^{k}(\sigma, \theta) \delta\left(\sigma-\sigma^{\prime}\right) \delta\left(\theta-\theta^{\prime}\right) .
\end{aligned}
$$

In order to introduce $R$-flux, we would like to consider a nontrivial restriction with twisting.

For simplicity, we assume that $\pi$ is nondegenerate. In order to obtain an AS type current algebra, we take the Liouville 1-form induced by the symplectic form $\boldsymbol{\omega}_{\mathcal{L}_{0}}$ defined by the Poisson bracket (7.32)-(7.34). This is $\alpha_{0}=-\int_{\mathcal{X}} \mu \boldsymbol{q}^{i}\left(\pi^{-1}\right)_{i j} \boldsymbol{d} \boldsymbol{x}^{j}+\cdots$, where $\cdots$ contains terms without $\boldsymbol{q}^{i}$.

Twisting by $\alpha_{0}$ induces the twist $\boldsymbol{p}_{i} \rightarrow \boldsymbol{p}_{i}-\left(\pi^{-1}\right)_{i j} \boldsymbol{d} \boldsymbol{x}^{j}$. After the restriction $\boldsymbol{\xi}_{i}=\boldsymbol{p}_{i}=0$ to the canonical Lagrangian submanifold, we get the Poisson brackets with $R$-flux,

$$
\begin{aligned}
& \left\{\boldsymbol{x}^{i}(\sigma, \theta), \boldsymbol{x}^{j}\left(\sigma^{\prime}, \theta^{\prime}\right)\right\}_{\mathrm{PB}}=0, \\
& \left\{\boldsymbol{x}^{i}(\sigma, \theta), \boldsymbol{q}^{j}\left(\sigma^{\prime}, \theta^{\prime}\right)\right\}_{\mathrm{PB}}=-\pi^{i j}(\boldsymbol{x}(\sigma, \theta)) \delta\left(\sigma-\sigma^{\prime}\right) \delta\left(\theta-\theta^{\prime}\right), \\
& \left\{\boldsymbol{q}^{i}(\sigma, \theta), \boldsymbol{q}^{j}\left(\sigma^{\prime}, \theta^{\prime}\right)\right\}_{\mathrm{PB}}=-\left(R^{i j k}(\boldsymbol{x})\left(\pi^{-1}\right)_{k l} \boldsymbol{d} \boldsymbol{x}^{l}+\frac{\partial \pi^{i j}}{\partial \boldsymbol{x}^{k}}(\boldsymbol{x}) \boldsymbol{q}^{k}\right) \delta\left(\sigma-\sigma^{\prime}\right) \delta\left(\theta-\theta^{\prime}\right) .
\end{aligned}
$$

Physical Poisson brackets are the degree zero components of these equations. Here we denote physical fields by $x^{i}(\sigma)=x^{(0) i}(\sigma)$ and $q^{i}(\sigma)=q^{(1) i}(\sigma)$. Then, the Poisson brackets on the physical canonical quantities are

$$
\begin{aligned}
& \left\{x^{i}(\sigma), x^{j}\left(\sigma^{\prime}\right)\right\}_{\mathrm{PB}}=0, \\
& \left\{x^{i}(\sigma), q^{j}\left(\sigma^{\prime}\right)\right\}_{\mathrm{PB}}=\pi^{i j}(x(\sigma)) \delta\left(\sigma-\sigma^{\prime}\right), \\
& \left\{q^{i}(\sigma), q^{j}\left(\sigma^{\prime}\right)\right\}_{\mathrm{PB}}=\left(-R^{i j k}(x)\left(\pi^{-1}\right)_{k l}(x) \partial_{\sigma} x^{l}+\frac{\partial \pi^{i j}}{\partial x^{k}}(x) q^{k}\right)(\sigma) \delta\left(\sigma-\sigma^{\prime}\right) .
\end{aligned}
$$

The relations (7.38)-(7.40) can also be derived by $\beta$-transformation. If $R=0$, the relations (7.38)-(7.40) are obtained by pullback of the Poisson structure on $T M$, lifted from the Poisson structure $\pi$ on $M$ [52], to the mapping space. In this case, the $R$ term is introduced by $\beta$-transformation, $x^{i} \rightarrow x^{i}$ and $q^{i} \rightarrow q^{i}+\beta^{i j} \pi_{j k}^{-1} \partial_{\sigma} x^{k}$, where $\beta=$ $\frac{1}{2} \beta^{i j}(x) \frac{\partial}{\partial x^{i}} \wedge \frac{\partial}{\partial x^{j}}$ is a bivector field such that $[\pi, \beta]_{S}=R$.

The symplectic form of Alekseev-Strobl type which induces (7.38)-(7.40) is

$$
\begin{aligned}
\omega= & \int_{S^{1}} d \sigma\left(\pi^{-1}\right)_{i j} \delta x^{i} \wedge \delta q^{j} \\
& -\frac{1}{2} \int_{S^{1}} d \sigma\left(-R^{i j k}\left(\pi^{-1}\right)_{k l} \partial_{\sigma} x^{l}+\frac{\partial \pi^{i j}}{\partial x^{k}} q^{k}\right)\left(\pi^{-1}\right)_{i m} \delta x^{m} \wedge\left(\pi^{-1}\right)_{j n} \delta x^{n} .
\end{aligned}
$$




\subsection{Contravariant current algebras with $R$-flux}

Here, currents are constructed from functions of degree equal to or less than one on the target space, $C_{0} \oplus C_{1}=\left\{f \in C^{\infty}\left(T^{*}[2] T[1] M\right)|| f \mid \leq 1\right\}$, which is the same space as in the case of the AS current algebra. Take a function of degree zero $j_{0}=f(x)$ and a function of degree one $j_{1}=X^{i}(x) p_{i}+\alpha_{i}(x) q^{i}$. By transgression of $j_{0}$ and $j_{1}$ to the mapping space, twisting by $\alpha_{0}$ such that $\boldsymbol{p}_{i} \rightarrow \boldsymbol{p}_{i}-\left(\pi^{-1}\right)_{i j} \boldsymbol{d} \boldsymbol{x}^{j}$, and restricting them to the canonical Lagrangian submanifold, we obtain the supergeometric currents,

$$
\begin{aligned}
\boldsymbol{J}_{(0)(f)}\left(\epsilon_{(1)}\right) & =\tilde{p r}{ }_{*} e^{\delta_{\alpha_{0}}} \mu_{*} \epsilon_{(1)} \mathrm{ev}^{*} j_{(0)(f)}=\int_{T[1] S^{1}} \mu \epsilon_{(1)} f(\boldsymbol{x}), \\
\boldsymbol{J}_{(1)(X+\alpha)}\left(\epsilon_{(0)}\right) & =\tilde{p r}{ }_{*} e^{\delta_{\alpha_{0}}} \mu_{*} \epsilon_{(0)} \mathrm{ev}^{*} j_{(1)(u, \alpha)}=\int_{T[1] S^{1}} \mu \epsilon_{(0)}\left(-X^{i}(\boldsymbol{x})\left(\pi^{-1}\right)_{i j} \boldsymbol{d} \boldsymbol{x}^{j}+\alpha_{i}(\boldsymbol{x}) \boldsymbol{q}^{i}\right) .
\end{aligned}
$$

If we take the degree zero components of the superfields, we obtain AS type currents,

$$
J_{0(f)}(\sigma)=f(x(\sigma)), \quad J_{1(X+\alpha)}(\sigma)=X^{i}(x(\sigma))\left(\pi^{-1}\right)_{i j} \partial_{\sigma} x^{j}(\sigma)+\alpha_{i}(x(\sigma)) q^{i}(\sigma) .
$$

The algebra of these supergeometric currents is computed from the Poisson brackets of the canonical quantities (7.35)-(7.37):

$$
\begin{aligned}
\left\{\boldsymbol{J}_{0(f)}(\epsilon), \boldsymbol{J}_{0(g)}\left(\epsilon^{\prime}\right)\right\}_{\mathrm{PB}}= & 0, \\
\left\{\boldsymbol{J}_{1(X+\alpha)}(\epsilon), \boldsymbol{J}_{0(g)}\left(\epsilon^{\prime}\right)\right\}_{\mathrm{PB}}= & \rho(X+\alpha) \boldsymbol{J}_{0(g)}\left(\epsilon \epsilon^{\prime}\right), \\
\left\{\boldsymbol{J}_{1(X+\alpha)}(\epsilon), \boldsymbol{J}_{1(Y+\beta)}\left(\epsilon^{\prime}\right)\right\}_{\mathrm{PB}}= & \boldsymbol{J}_{1\left([X+\alpha, Y+\beta]_{R}^{\pi}\left(\epsilon \epsilon^{\prime}\right)\right.} \\
& +\int_{T[1] S^{1}} \mu \boldsymbol{d} \epsilon_{(0)} \epsilon_{(0)}^{\prime}\langle X+\alpha, Y+\beta\rangle(\boldsymbol{x}),
\end{aligned}
$$

where

$$
[X+\alpha, Y+\beta]_{R}^{\pi}=[\alpha, \beta]_{\pi}+L_{\alpha}^{\pi} Y-\iota_{\beta} d_{\pi} X-R(\alpha, \beta,-),
$$

is the contravariant Dorfman bracket with $R$-flux on $T M \oplus T^{*} M$ and $\rho(X+\alpha)=\pi^{\sharp}(\alpha)$ is the anchor map. Component expansions give rise to physical current algebras:

$$
\begin{aligned}
\left\{J_{0(f)}(\sigma), J_{0(g)}\left(\sigma^{\prime}\right)\right\}_{\mathrm{PB}}= & 0 \\
\left\{J_{1(X+\alpha)}(\sigma), J_{0(g)}\left(\sigma^{\prime}\right)\right\}_{\mathrm{PB}}= & -\rho(X+\alpha) J_{0(g)}(x(\sigma)) \delta\left(\sigma-\sigma^{\prime}\right), \\
\left\{J_{1(X+\alpha)}(\sigma), J_{1(Y+\beta)}\left(\sigma^{\prime}\right)\right\}_{\mathrm{PB}}= & -J_{1\left([X+\alpha, Y+\beta]_{R}^{\pi}\right)}(\sigma) \delta\left(\sigma-\sigma^{\prime}\right) \\
& +\langle X+\alpha, Y+\beta\rangle\left(\sigma^{\prime}\right) \partial_{\sigma} \delta\left(\sigma-\sigma^{\prime}\right) .
\end{aligned}
$$

This formula (7.45)-(7.47) is consistent, even if the Poisson structure $\pi$ is degenerate. Therefore, we do not need to impose a nondegeneracy condition for $\pi$ in the current algebra.

\section{Conclusions and discussion}

The Poisson Courant algebroid, which is a contravariant object of the standard Courant algebroid, has been formulated by supergeometric construction. The duality between these 
two specific Courant algebroids has been analyzed in detail. As a result, the duality transformation is a canonical transformation on the graded symplectic manifold and the transformation between the 3 -form $H$-flux in the standard Courant algebroid and the trivector $R$-flux in the Poisson Courant algebroid has been derived. In [29, 48, 53], twisting of a bivector field by a 3-form $H$, a so-called twisted Poisson structure, has been discussed. From the above duality, we have obtained its contravariant geometric structure in (6.17), twisting of a 2 -form by a trivector field $R$.

Moreover, we have shown that this duality is, from the mathematical viewpoint, the generalization of the correspondence between the de Rham cohomology and the Poisson cohomology. We also discussed that the same duality can be derived on the sigma model level.

By using the supergeometric formulation, we have constructed a 3-dimensional AKSZ sigma model and a 2-dimensional boundary sigma model with the structure of a Poisson Courant algebroid. From the physical viewpoint, we are considering a theory of a topological membrane on a Poisson manifold. From the general form of the homological function given in (3.11), (see also [28, 29]) we can introduce all types of third rank tensors $(H, F, Q$ and $R$ ) with various covariant and contravariant suffixes. However, it is remarkable that the 3 -vector $R$ can only be introduced on the Poisson manifold. Then, we derived the topological string on the Poisson manifold as the boundary theory of that topological membrane. Of course, the theory obtained in this way can be identified with the Poisson sigma model by field redefinition. What we found is that there is a specific way to lift the boundary theory to the topological membrane including $R$-flux on the Poisson manifold.

We have also constructed a current algebra with $R$-flux on the tangent space of the loop space from the target space QP-manifold data. The resulting current algebra is the contravariant counterpart of the current algebra with $H$-flux of Alekseev-Strobl type.

The $R$-flux has also been discussed in $[54,55]$ using double field theory. There, the nongeometric $R$-flux is characterized as a Jacobiator, the quantity corresponding to the anomaly of the Jacobi identity, i.e. $R^{i j k} \sim \beta^{l[i} \frac{\partial \beta^{j k]}}{\partial x^{l}}$. In section 5 , we have discussed the Poisson Courant algebroid and its trivector field $R$ from the point of view of double field theory. If we take the special solution of the section condition defined by the Poisson structure $\pi$, the resulting spacetime has the Poisson Courant algebroid structure. Therefore, we have found that our formalism describes the $R$-flux in the frame specified by this particular solution.

In our formulation, $\beta$ is independent of the Poisson bivector $\pi$, thus we can consider the special case $[\pi+\beta, \pi+\beta]_{S}=0$. It means that $\pi+\beta$ is again a Poisson structure. Note that it does not mean a deformation of the Poisson Courant algebroid. This is a Maurer-Cartan condition of $\beta, d_{\pi} \beta+\frac{1}{2}[\beta, \beta]_{S}=0$, and we obtain the $R$-flux as the Jacobiator $[56,57]$,

$$
R=-\frac{1}{2}[\beta, \beta]_{S}
$$

This is the same formula in the definition inspired by the double field theory. The meaning of this observation will be discussed in future work. 


\section{Acknowledgments}

The authors would like to thank T. Asakawa, B. Jurčo, Y. Kaneko, H. Muraki, Y. Maeda and U. Carow-Watamura for helpful discussions and valuable comments. M.A. Heller is supported by Japanese Government (MONBUKAGAKUSHO) Scholarship and N. Ikeda is supported by the research promotion program grant at Ritsumeikan University.

\section{A Formulas in graded differential calculus}

We summarize formulas of graded symplectic geometry.

\section{A.1 Basic definitions}

Let $z$ be a local coordinate on a graded manifold $\mathcal{M}$. A differential on a function is defined by

$$
d f(z)=d z^{a} \frac{\vec{\partial} f}{\partial z^{a}}
$$

A vector field $X$ is expanded using local coordinates by

$$
X=X^{a}(z) \frac{\vec{\partial}}{\partial z^{a}}
$$

The interior product is defined by the differentiation by the following graded vector field on $T[1] \mathcal{M}$,

$$
\iota_{X}=(-1)^{|X|} X^{a}(z) \frac{\vec{\partial}}{\partial d z^{a}}
$$

where we define $\frac{\vec{\partial}}{\partial d z^{a}} d z^{b}=\delta^{b}{ }_{a}$. For a graded differential form $\alpha$, we denote $|\alpha|$ as total degree (form degree plus degree by grading) of $\alpha$. Note that $|d|=1,\left|d z^{a}\right|=\left|z^{a}\right|+1$ and $\left|\iota_{X}\right|=|X|-1$. For vector fields, $X=X^{a}(z) \frac{\vec{\partial}}{\partial z^{a}}, Y=Y^{a}(z) \frac{\vec{\partial}}{\partial z^{a}}$, the graded Lie bracket is

$$
[X, Y]=X^{a} \frac{\vec{\partial} Y^{b}}{\partial z^{a}} \frac{\vec{\partial}}{\partial z^{b}}-(-1)^{|X||Y|} Y^{a} \frac{\vec{\partial} X^{b}}{\partial z^{a}} \frac{\vec{\partial}}{\partial z^{b}} .
$$

We obtain the following formula,

$$
X f=(-1)^{|X|} \iota_{X} d f=(-1)^{(|f|+1)|X|} d f(X),
$$

where

$$
d z^{a}\left(\frac{\vec{\partial}}{\partial z^{b}}\right)=\delta_{b}^{a}
$$

Proof. We prove (A.5). Since $X f=X^{a}(z) \frac{\vec{\partial} f}{\partial z^{a}}$, we have

$$
(-1)^{|X|} \iota_{X} d f=(-1)^{|X|}(-1)^{|X|} X^{a}(z) \frac{\vec{\partial}}{\partial d z^{a}}\left(d z^{a} \frac{\vec{\partial} f}{\partial z^{a}}\right) .
$$


Therefore,

$$
\begin{aligned}
& d f(X)=d z^{a} \frac{\vec{\partial} f}{\partial z^{a}}\left(X^{b}(z) \frac{\vec{\partial}}{\partial z^{b}}\right) \\
&=(-1)^{(|f|-|z|)|X|}\left[d z^{a}\left(X^{b}(z) \frac{\vec{\partial}}{\partial z^{b}}\right)\right] \overrightarrow{\frac{\partial}{\partial z^{a}}} \\
&=(-1)^{(|f|-|z|)|X|}(-1)^{(|X|-|z|)(|z|+1)} X^{b}(z)\left[d z^{a}\left(\frac{\vec{\partial}}{\partial z^{b}}\right)\right] \vec{\partial} f \\
& \partial z^{a}
\end{aligned}
$$

\section{A.2 Cartan formulas}

The Lie derivative is defined by

$$
L_{X}=\iota_{X} d-(-1)^{(|X|-1) \times 1} d \iota_{X}=\iota_{X} d+(-1)^{|X|} d \iota_{X} .
$$

Its degree is $\left|L_{X}\right|=|X|$.

Let $\alpha$ and $\beta$ be graded differential forms. We can show the following graded Cartan formulas,

$$
\begin{aligned}
\alpha \wedge \beta & =(-)^{|\alpha||\beta|} \beta \wedge \alpha, \\
d(\alpha \wedge \beta) & =d \alpha \wedge \beta+(-1)^{|\alpha|} \alpha \wedge d \beta, \\
\iota_{X}(\alpha \wedge \beta) & =\iota_{X} \alpha \wedge \beta+(-1)^{|\alpha|(|X|+1)} \alpha \wedge \iota_{X} \beta, \\
L_{X}(\alpha \wedge \beta) & =L_{X} \alpha \wedge \beta+(-1)^{|\alpha||X|} \alpha \wedge L_{X} \beta, \\
L_{X} d & =(-1)^{|X|} d L_{X}, \\
\iota_{X} \iota_{Y}-(-1)^{(|X|-1)(|Y|-1)} \iota_{Y} \iota_{X} & =0, \\
L_{X} \iota_{Y}-(-1)^{|X|(|Y|-1)} \iota_{Y} L_{X} & =\iota_{[X, Y]}, \\
L_{X} L_{Y}-(-1)^{|X||Y|} L_{Y} L_{X} & =L_{[X, Y]} .
\end{aligned}
$$

\section{A.3 Differential forms}

Let $\alpha=d z^{a_{1}} \wedge \cdots \wedge d z^{a_{m}} \alpha_{a_{1} \cdots a_{m}}(z)$ be an $m$-form on $\mathcal{M}$. The contraction of $\alpha(X,-, \cdots,-)$ with a vector field $X$ on $\mathcal{M}$ is

$$
\alpha(X,-, \cdots,-)=(-1)^{|X|(|\alpha|+1)} \iota_{X} \alpha(-, \cdots,-) .
$$

Proof.

$$
\begin{aligned}
\alpha(X,-, \cdots,-) & =d z^{a_{1}} \wedge \cdots \wedge d z^{a_{m}} \alpha_{a_{1} \cdots a_{m}}(z)\left(X^{b} \frac{\vec{\partial}}{\partial z^{b}}\right) \\
& =(-1)^{|X|(|\alpha|-|z|-1)} d z^{a_{1}}\left(X^{b} \frac{\vec{\partial}}{\partial z^{b}}\right) d z^{a_{2}} \wedge \cdots \wedge d z^{a_{m}} \alpha_{a_{1} \cdots a_{m}}(z)
\end{aligned}
$$




$$
\begin{aligned}
& =(-1)^{|X|(|\alpha|-|z|-1)}(-1)^{(|X|-|z|)(|z|+1)} X^{a_{1}} d z^{a_{2}} \wedge \cdots \wedge d z^{a_{m}} \alpha_{a_{1} \cdots a_{m}}(z) \\
& =(-1)^{|X||\alpha|} X^{a_{1}} d z^{a_{2}} \wedge \cdots \wedge d z^{a_{m}} \alpha_{a_{1} \cdots a_{m}}(z) \\
& =(-1)^{|X||\alpha|}(-1)^{|X|} \iota_{X} \alpha
\end{aligned}
$$

By induction using (A.18), we obtain the general formula,

$$
\begin{aligned}
\alpha\left(X_{m}, X_{m-1}, \cdots, X_{1}\right) & =-(-1)^{\sum_{i=1}^{m}\left|X_{i}\right|(|\alpha|+i)} \iota_{X_{m}} \cdots \iota_{X_{1}} \alpha \\
\alpha\left(X_{m}, \cdots, X_{j}, \cdots, X_{i}, \cdots X_{1}\right) & =-(-1)^{\left|X_{i}\right|\left|X_{j}\right|} \alpha\left(X_{m}, \cdots, X_{i}, \cdots, X_{j}, \cdots X_{1}\right) .
\end{aligned}
$$

Especially, if $\alpha$ is a 2-form, we derive

$$
\alpha(X, Y)=-(-1)^{|X||Y|} \alpha(Y, X) .
$$

\section{A.3.1 Exterior derivatives}

The exterior derivative on a function is given by (A.5),

$$
d f(X)=(-1)^{|X|(|f|+1)} X f .
$$

Let $\alpha$ be a 1 -form on $\mathcal{M}$. Then, from the Cartan formulas, we obtain

$$
d \alpha\left(X_{1}, X_{2}\right)=(-1)^{\left|X_{1}\right||\alpha|} X_{1} \alpha\left(X_{2}\right)-(-1)^{\left|X_{2}\right||\alpha|}(-1)^{\left|X_{1}\right|\left|X_{2}\right|} X_{2} \alpha\left(X_{1}\right)-\alpha\left(\left[X_{1}, X_{2}\right]\right) .
$$

For a 2-form $\alpha$, the formula gives

$$
\begin{aligned}
d \alpha\left(X_{1}, X_{2}, X_{3}\right)= & (-1)^{\left|X_{1}\right|(|\alpha|+1)} X_{1} \alpha\left(X_{2}, X_{3}\right)-(-1)^{\left|X_{2}\right|(|\alpha|+1)}(-1)^{\left|X_{1}\right|\left|X_{2}\right|} X_{2} \alpha\left(X_{1}, X_{3}\right) \\
& +(-1)^{\left|X_{3}\right|(|\alpha|+1)}(-1)^{\left(\left|X_{1}\right|+\left|X_{2}\right|\right)\left|X_{3}\right|} X_{3} \alpha\left(X_{1}, X_{2}\right)-\alpha\left(\left[X_{1}, X_{2}\right], X_{3}\right) \\
& +(-1)^{\left|X_{2}\right|\left|X_{3}\right|} \alpha\left(\left[X_{1}, X_{3}\right], X_{2}\right)-(-1)^{\left|X_{1}\right|\left(\left|X_{2}\right|+\left|X_{3}\right|\right)} \alpha\left(\left[X_{2}, X_{3}\right], X_{1}\right) .
\end{aligned}
$$

Let $\alpha=d z^{a_{1}} \wedge \cdots \wedge d z^{a_{m}} \alpha_{a_{1} \cdots a_{m}}(z)$ be an $m$-form on $\mathcal{M}$. Then, we can prove the following formula by induction,

$$
\begin{aligned}
d \alpha & \left(X_{1}, X_{2}, \cdots, X_{m}\right) \\
= & \sum_{i=1}^{m}(-1)^{i-1}(-1)^{\left|X_{i}\right|(|\alpha|+m)}(-1)^{\sum_{k=1}^{i-1}\left|X_{i}\right|\left|X_{k}\right|} X_{i} \alpha\left(X_{1}, \cdots, \hat{X}_{i}, \cdots, X_{m}\right) \\
& +\sum_{i<j}(-1)^{i+j}(-1)^{\sum_{k=1}^{i-1}\left|X_{i}\right|\left|X_{k}\right|+\sum_{l=1, l \neq j}^{j-1}\left|X_{j}\right|\left|X_{l}\right|} \alpha\left(\left[X_{i}, X_{j}\right], \cdots, \hat{X}_{i}, \cdots, \hat{X}_{j}, \cdots, X_{m}\right) .
\end{aligned}
$$

\section{A.4 Graded symplectic form and Poisson bracket}

Let $\omega$ be a symplectic form of degree $n$. Since $\omega$ is a 2 -form, its total degree is $|\omega|=n+2$. Let $z=\left(q^{a}, p_{a}\right)$ be Darboux coordinates such that $|q|+|p|=n$. Then we obtain

$$
\begin{aligned}
\omega & =(-1)^{|q|(|p|+1)} d q^{a} \wedge d p_{a}=(-1)^{n|q|} d q^{a} \wedge d p_{a} \\
& =(-1)^{n|q|}(-1)^{(|q|+1)(|p|+1)} d p_{a} \wedge d q^{a}=(-1)^{|p|+1} d p_{a} \wedge d q^{a} .
\end{aligned}
$$


Then, the Liouville 1 -form $\omega=-d \vartheta$ is given by

$$
\begin{aligned}
\vartheta & =(-1)^{|p|} p_{a} d q^{a}=-(-1)^{n+1-|q|} p_{a} d q^{a}=(-1)^{|q||p|} d q^{a} p_{a} \\
& =-(-1)^{|q|(|p|+1)} q^{a} d p_{a}=-d p_{a} q^{a} .
\end{aligned}
$$

The Hamiltonian vector field $X_{f}$ of a function $f$ is defined by

$$
\iota_{X_{f}} \omega=-d f .
$$

Its total degree is $\left|X_{f}\right|=|f|-n$. In order to obtain the Darboux coordinate expression of $X_{f}$, assume that $X=X_{a} \frac{\vec{\partial}}{\partial p_{a}}+Y^{a} \frac{\vec{\partial}}{\partial q^{a}}$. Then we derive

$$
\begin{aligned}
\iota_{X_{f}} \omega & =\left((-1)^{|X|+p} X_{a} \frac{\vec{\partial}}{\partial d p_{a}}+(-1)^{|X|+q} Y^{a} \frac{\vec{\partial}}{\partial d q^{a}}\right) \cdot\left((-1)^{n|q|} d q^{a} \wedge d p_{a}\right) \\
& =-d q^{a} \frac{\vec{\partial} f}{\partial q^{a}}-d p_{a} \frac{\vec{\partial} f}{\partial p_{a}} .
\end{aligned}
$$

By solving this equation, we finally obtain

$$
X_{f}=\frac{f \overleftarrow{\partial}}{\partial q^{a}} \frac{\vec{\partial}}{\partial p_{a}}-(-1)^{|q||p|} \frac{f \overleftarrow{\partial}}{\partial p_{a}} \frac{\vec{\partial}}{\partial q^{a}}
$$

Here, $\frac{f \overleftarrow{\partial}}{\partial q^{a}}=(-1)^{(|f|-q) q} \frac{\vec{\partial} f}{\partial q^{a}}$ is the right derivative.

The graded Poisson bracket is defined by

$$
\{f, g\}=X_{f} g=(-1)^{|f|+n} \iota_{X_{f}} d g=(-1)^{|f|+n+1} \iota_{X_{f}} \iota_{X_{g}} \omega .
$$

It satisfies

$$
\begin{aligned}
\{f, g\} & =-(-1)^{(|f|-n)(|g|-n)}\{g, f\}, \\
\{f, g h\} & =\{f, g\} h+(-1)^{(|f|-n)|g|} g\{f, h\}, \\
\{f,\{g, h\}\} & =\{\{f, g\}, h\}+(-1)^{(|f|-n)(|g|-n)}\{g,\{f, h\}\} .
\end{aligned}
$$

For the Darboux coordinates, we get the relations

$$
\left\{q^{a}, p_{b}\right\}=\delta_{b}^{a}, \quad\left\{p_{b}, q^{a}\right\}=-(-1)^{|q||p|} \delta_{b}^{a} .
$$

For functions, $f=f(q, p)$ and $g=g(q, p)$, the graded Poisson bracket is given by

$$
\{f, g\}=\frac{f \overleftarrow{\partial}}{\partial q^{a}} \frac{\vec{\partial} g}{\partial p_{a}}-(-1)^{|q||p|} \frac{f \overleftarrow{\partial}}{\partial p_{a}} \frac{\vec{\partial} g}{\partial q^{a}}
$$

$X$ is called symplectic vector field, if $L_{X} \omega=0$, i.e., $d \iota_{X} \omega=0$. Let $X, Y$ be symplectic vector fields. Then, $[X, Y]$ is the Hamiltonian vector field for $-(-1)^{|X|} \iota_{X} \iota_{Y} \omega$.

Proof.

$$
\begin{aligned}
\iota_{[X, Y]} \omega & =\left(L_{X} \iota_{Y}-(-1)^{|X|(|Y|-1)} \iota_{Y} L_{X}\right) \omega=(-1)^{|X|} d \iota_{X} \iota_{Y} \omega \\
& =-d\left[-(-1)^{|X|} \iota_{X} \iota_{Y} \omega\right] .
\end{aligned}
$$


If $X=X_{f}, Y=X_{g}$ are Hamiltonian vector fields, then the following equation holds,

$$
\iota_{\left[X_{f}, X_{g}\right]} \omega=(-1)^{|f|+n} d \iota_{X_{f}} \iota_{X_{g}} \omega .
$$

Therefore, we get

$$
X_{\{f, g\}}=-\left[X_{f}, X_{g}\right]
$$

Since $\iota_{X_{f}} \iota_{X_{g}} \omega=-(-1)^{|f| n+|g|(n+1)} \omega\left(X_{g}, X_{f}\right)$, we easily derive

$$
\begin{aligned}
\{f, g\} & =(-1)^{|f|+n+1} \iota_{X_{f}} \iota_{X_{g}} \omega \\
& =(-1)^{(|f|+|g|)(n+1)} \omega\left(X_{g}, X_{f}\right)=(-1)^{|f||g|+n+1} \omega\left(X_{f}, X_{g}\right) .
\end{aligned}
$$

We consider the AKSZ construction on $\operatorname{Map}(\mathcal{X}, \mathcal{M})$. Let $D$ be a differential on $\mathcal{X}$. It can be locally expressed as $D=\theta^{\mu} \frac{\partial}{\partial \sigma^{\mu}}$. We denote with $\hat{D}$ the vector field on $\operatorname{Map}(\mathcal{X}, \mathcal{M})$ of degree 1 , which is induced by $D$. Then the following equation holds,

$$
\left\{\iota_{\hat{D}} \mu_{*} \mathrm{ev}^{*} \vartheta, \mu_{*} \mathrm{ev}^{*} f\right\}=-\iota_{\hat{D}} \mu_{*} \mathrm{ev}^{*} d f\left(=\int d^{n+1} \sigma d^{n+1} \theta \boldsymbol{d} f(\sigma, \theta)\right),
$$

for $f \in C^{\infty}(\mathcal{M})$.

Proof. $S_{0}=\iota_{\hat{D}} \mu_{*} \mathrm{ev}^{*} \vartheta$ is a Hamiltonian for the vector field $\hat{D}$,i.e., $X_{S_{0}}=\hat{D}$. Therefore, we get

$$
\begin{aligned}
\left\{\iota_{\hat{D}} \mu_{*} \mathrm{ev}^{*} \vartheta, \mu_{*} \mathrm{ev}^{*} f\right\} & =\left\{S_{0}, \mu_{*} \mathrm{ev}^{*} f\right\} \\
& =(-1)^{\left|S_{0}\right|} \iota^{\iota} \iota^{\iota} X_{\mu_{*} \mathrm{ev}_{f}} \boldsymbol{\omega} \\
& =-\iota_{\hat{D}} \mu_{*} \mathrm{ev}^{*} d f .
\end{aligned}
$$

\section{B Formulas on the mapping space}

\section{B.1 Functional differential calculus}

We list the formulas, that we use on the mapping $\operatorname{space} \operatorname{Map}(\mathcal{X}, \mathcal{M})$. Let $X$ be a manifold of dimension $d=n+1$. The mapping space functions are superfields. They depend on variables on $\mathcal{X}=T[1] X$, which is a $(d, d)$-dimensional supermanifold with even local coordinates $\sigma^{\mu}$ and odd local coordinates $\theta^{\mu}$, where $\mu=1, \ldots, d$.

A component expansion of a superfield $\Phi(\sigma, \theta)$ of degree $|\Phi|$ in Grassmann variables is defined as

$$
\Phi(\sigma, \theta)=\sum_{j=0}^{d} \frac{1}{j !} \phi_{\mu_{1} \cdots \mu_{j}}(\sigma) \theta^{\mu_{1}} \cdots \theta^{\mu_{j}},
$$

where the $j=0$ term $\phi(\sigma)$ is not accompanied by $\theta^{\mu}$. Since $\left|\theta^{\mu}\right|=1$, we get $\left|\phi_{\mu_{1} \cdots \mu_{j}}\right|=$ $|\Phi|-j$. 
The functional derivative on the mapping space is

$$
\frac{\vec{\delta} \Phi(\sigma, \theta)}{\delta \Phi\left(\sigma^{\prime}, \theta^{\prime}\right)}=\delta^{d}\left(\sigma^{\prime}-\sigma\right) \delta^{d}\left(\theta^{\prime}-\theta\right)
$$

Expanding this equation in components, we obtain the formula for the left functional derivative,

$$
\frac{\vec{\delta}}{\delta \Phi(\sigma, \theta)}=\sum_{j=0}^{d} \frac{(-1)^{d-j}}{j !(d-j) !} \theta^{\mu_{1}} \cdots \theta^{\mu_{j}} \epsilon_{\mu_{1} \cdots \mu_{j} \mu_{j+1} \cdots \mu_{d}} \frac{\vec{\delta}}{\delta \phi_{\mu_{j+1} \cdots \mu_{d}}(\sigma)}
$$

where $\epsilon_{\mu_{1} \cdots \mu_{j} \mu_{j+1} \cdots \mu_{d}}$ is the completely antisymmetric Levi-Civita symbol. By degree counting, we obtain $\left|\frac{\vec{\delta}}{\delta \phi_{\mu_{j+1} \cdots \mu_{d}}(\sigma)}\right|=-(|\Phi|-d+j)$. We require the following identity for the right functional derivative,

$$
\frac{\vec{\delta} F}{\delta \Phi}=(-1)^{|F|(|\Phi|-d)} \frac{F \overleftarrow{\delta}}{\delta \Phi},
$$

for an arbitrary superfield $F$. From this equation, we have the right derivative for components,

$$
\frac{\overleftarrow{\delta}}{\delta \Phi(\sigma, \theta)}=\sum_{j=0}^{d} \frac{1}{j !(d-j) !}(-1)^{|\Phi|+j(|\Phi|+d+1)} \frac{\overleftarrow{\delta}}{\delta \phi_{\mu_{j+1} \cdots \mu_{d}}(\sigma)} \theta^{\mu_{1}} \cdots \theta^{\mu_{j}} \epsilon_{\mu_{1} \cdots \mu_{j} \mu_{j+1} \cdots \mu_{d}}
$$

Computing the right derivative $\frac{\Phi(\sigma, \theta) \overleftarrow{\delta}}{\delta \Phi\left(\sigma^{\prime}, \theta^{\prime}\right)}$ using the above formula, we summarize

$$
\begin{aligned}
& \frac{\vec{\delta} \Phi(\sigma, \theta)}{\delta \Phi\left(\sigma^{\prime}, \theta^{\prime}\right)}=\delta^{d}\left(\sigma^{\prime}-\sigma\right) \delta^{d}\left(\theta^{\prime}-\theta\right) \\
& \frac{\Phi(\sigma, \theta) \overleftarrow{\delta}}{\delta \Phi\left(\sigma^{\prime}, \theta^{\prime}\right)}=(-1)^{|\Phi|(1+d)+d} \delta^{d}\left(\sigma-\sigma^{\prime}\right) \delta^{d}\left(\theta-\theta^{\prime}\right)
\end{aligned}
$$

The degrees of the right and left derivatives are

$$
\left|\frac{\vec{\delta}}{\delta \Phi(\sigma, \theta)}\right|=\left|\frac{\overleftarrow{\delta}}{\delta \Phi(\sigma, \theta)}\right|=d-|\Phi|
$$

For an arbitrary superfield $F$, we have

$$
\left|\frac{\vec{\delta} F}{\delta \Phi(\sigma, \theta)}\right|=\left|\frac{F \overleftarrow{\delta}}{\delta \Phi(\sigma, \theta)}\right|=|F|+d-|\Phi|
$$

The following left and right Leibniz rules hold for arbitrary superfields $F$ and $G$ :

$$
\begin{aligned}
& \frac{\vec{\delta}}{\delta \Phi}(F G)=\frac{\vec{\delta} F}{\delta \Phi} \cdot G+(-1)^{|F|(d-|\Phi|)} F \cdot \frac{\vec{\delta} G}{\delta \Phi} \\
& (F G) \frac{\overleftarrow{\delta}}{\delta \Phi}=F \cdot \frac{G \overleftarrow{\delta}}{\delta \Phi}+(-1)^{|G|(d-|\Phi|)} \frac{F \overleftarrow{\delta}}{\delta \Phi} \cdot G
\end{aligned}
$$

The measure on the worldvolume supermanifold is defined by $\mu=d \sigma^{1} \cdots d \sigma^{d} d \theta^{d} \cdots d \theta^{1}$ and its degree is $|\mu|=-d$. The following equation for the Grassmann delta function holds

$$
\int \mu_{\theta} \delta^{d}\left(\theta-\theta^{\prime}\right) \Phi(\sigma, \theta)=\Phi\left(\sigma, \theta^{\prime}\right)
$$

where $\mu_{\theta}=d^{d} \theta \cdots d^{1} \theta$. 


\section{B.2 Graded symplectic geometry}

In this subsection, we map the structures on $\mathcal{M}$ to structures on the target space $\operatorname{Map}(\mathcal{X}, \mathcal{M})$ by the transgression map $\mu_{*} \mathrm{ev}^{*}$.

Let $\boldsymbol{z}^{i}(\sigma, \theta)$ be a local basis superfield of the mapping space $\operatorname{Map}(\mathcal{X}, \mathcal{M})$, corresponding to a local coordinate $z^{i}$ on $\mathcal{M}$. We write a vector field on the mapping space for $X=$ $X^{i}(z) \frac{\vec{\partial}}{\partial z^{i}}$ as

$$
X=\int_{\mathcal{X}} \mu(-1)^{d\left|X^{i}\right|} X^{i}(\boldsymbol{z}(\sigma, \theta)) \frac{\vec{\delta}}{\delta \boldsymbol{z}^{i}(\sigma, \theta)} .
$$

Then, the interior product is

$$
\iota_{X}=(-1)^{|X|} \int_{\mathcal{X}} \mu(-1)^{d\left|X^{i}\right|} X^{i}(\boldsymbol{z}(\sigma, \theta)) \frac{\vec{\delta}}{\delta\left(\delta \boldsymbol{z}^{i}\right)(\sigma, \theta)} .
$$

The symplectic form $\boldsymbol{\omega}$ on the mapping space corresponding to $\omega=(-1)^{n|q|} \delta q^{i} \wedge \delta p_{i}$ is defined as

$$
\boldsymbol{\omega}=\int_{\mathcal{X}} \mu(-1)^{(d-1)|q|} \delta \boldsymbol{q}^{i}(\sigma, \theta) \wedge \delta \boldsymbol{p}_{i}(\sigma, \theta) .
$$

We have $|\boldsymbol{\omega}|=|\mu|+1+\left|\boldsymbol{q}^{i}\right|+1+\left|\boldsymbol{p}_{i}\right|=-d+2+d-1=1 .^{7}$

The differential on a function $f$ is

$$
\boldsymbol{\delta} f=\int_{\mathcal{X}} \mu(-1)^{d\left(\left|\boldsymbol{z}^{i}\right|+1\right)}\left(\delta \boldsymbol{z}^{i}\right)(\sigma, \theta) \frac{\vec{\delta} f}{\delta \boldsymbol{z}^{i}(\sigma, \theta)} .
$$

We define the Liouville 1-form (the canonical 1-form) $\vartheta$ on the mapping space as

$$
\boldsymbol{\omega}=-\boldsymbol{\delta \vartheta} .
$$

The Hamiltonian vector field is defined by

$$
\iota_{X_{f}} \boldsymbol{\omega}=-\boldsymbol{\delta} f
$$

and the $B V$ bracket we define by

$$
\{f, g\}_{\mathrm{BV}}=X_{f} g .
$$

Then, direct computation gives the following local expression of the BV bracket on the mapping space,

$$
\{f, g\}_{\mathrm{BV}}=(-1)^{d-|q|} \int_{\mathcal{X}}\left[\frac{f \overleftarrow{\delta}}{\delta \boldsymbol{q}^{i}} \mu \frac{\vec{\delta} g}{\delta \boldsymbol{p}_{i}}+(-1)^{d(1+|q|)} \frac{f \overleftarrow{\delta}}{\delta \boldsymbol{p}_{i}} \mu \frac{\vec{\delta} g}{\delta \boldsymbol{q}^{i}}\right]
$$

We can prove the following identities of the graded Poisson bracket of degree 1,

$$
\begin{aligned}
\{f, g\}_{\mathrm{BV}} & =-(-1)^{(|f|+1)(|g|+1)}\{g, f\}_{\mathrm{BV}} \\
\{f, g h\}_{\mathrm{BV}} & =\{f, g\}_{\mathrm{BV}} h+(-1)^{(|f|+1)|g|} g\{f, h\}_{\mathrm{BV}}, \\
\left\{f,\{g, h\}_{\mathrm{BV}}\right\}_{\mathrm{BV}} & =\left\{\{f, g\}_{\mathrm{BV}}, h\right\}_{\mathrm{BV}}+(-1)^{(|f|+1)(|g|+1)}\left\{g,\{f, h\}_{\mathrm{BV}}\right\}_{\mathrm{BV}} .
\end{aligned}
$$

\footnotetext{
${ }^{7}$ This is degree counting to determine the sign factor. In fact, $\boldsymbol{\omega}$ is a 2 -form of degree $d-1$. Then we count the sign factor as degree $d+1$.
} 
Remark B.1. We list up the degrees of the defined objects.

A vector field has $|X|=-d+\left|X^{i}\right|+d-\left|z^{i}\right|=\left|X^{i}\right|-\left|z^{i}\right|$. An interior product has $\left|\iota_{X}\right|=-d+\left|X^{i}\right|+d-\left(\left|\boldsymbol{z}^{i}\right|+1\right)=\left|X^{i}\right|-\left|z^{a}\right|-1=|X|-1$, since $\left|\delta \boldsymbol{z}^{i}\right|=\left|\boldsymbol{z}^{i}\right|+1$. A symplectic structure has $|\boldsymbol{\omega}|=-d+1+\left|\boldsymbol{q}^{i}\right|+1+\left|\boldsymbol{p}_{i}\right|=1$. A differential on a function has $|\boldsymbol{\delta} f|=-d+1+\left|\boldsymbol{z}^{i}\right|+|f|+d-\left|\boldsymbol{z}^{i}\right|=1+|f|$. A Hamiltonian vector field has $\left|X_{f}\right|=1+|f|$ and therefore $\left|\iota_{X_{f}}\right|=|f|$.

Open Access. This article is distributed under the terms of the Creative Commons Attribution License (CC-BY 4.0), which permits any use, distribution and reproduction in any medium, provided the original author(s) and source are credited.

\section{References}

[1] C.M. Hull, A Geometry for non-geometric string backgrounds, JHEP 10 (2005) 065 [hep-th/0406102] [INSPIRE].

[2] J. Shelton, W. Taylor and B. Wecht, Nongeometric flux compactifications, JHEP 10 (2005) 085 [hep-th/0508133] [INSPIRE].

[3] M. Graña, R. Minasian, M. Petrini and D. Waldram, T-duality, generalized geometry and non-geometric backgrounds, JHEP 04 (2009) 075 [arXiv:0807.4527] [INSPIRE].

[4] C. Hull and B. Zwiebach, Double field theory, JHEP 09 (2009) 099 [arXiv: 0904.4664] [INSPIRE].

[5] J. de Boer and M. Shigemori, Exotic branes in string theory, Phys. Rept. 532 (2013) 65 [arXiv: 1209.6056] [INSPIRE].

[6] F. Hassler and D. Lüst, Non-commutative/non-associative IIA (IIB) Q- and R-branes and their intersections, JHEP 07 (2013) 048 [arXiv:1303.1413] [INSPIRE].

[7] D. Andriot and A. Betz, $\beta$-supergravity: a ten-dimensional theory with non-geometric fluxes and its geometric framework, JHEP 12 (2013) 083 [arXiv:1306.4381] [INSPIRE].

[8] P. Bouwknegt, J. Evslin and V. Mathai, T duality: topology change from H flux, Commun. Math. Phys. 249 (2004) 383 [hep-th/0306062] [INSPIRE].

[9] P. Bouwknegt, K. Hannabuss and V. Mathai, T-duality for principal torus bundles and dimensionally reduced Gysin sequences, Adv. Theor. Math. Phys. 9 (2005) 749 [hep-th/0412268] [INSPIRE].

[10] P. Bouwknegt, Lectures on cohomology, T-duality and generalized geometry, Lect. Notes Phys. 807 (2010) 261 [INSPIRE].

[11] T. Courant, Dirac manifolds, Trans. Amer. Math. Soc. 319 (1990) 631.

[12] Z.-J. Liu, A. WEinstein and P. Xu, Manin triples for Lie bialgebroids, J. Diff. Geom. 45 (1997) 547 [dg-ga/9508013] [INSPIRE].

[13] N. Hitchin, Generalized Calabi-Yau manifolds, Quart. J. Math. 54 (2003) 281 [math/0209099] [INSPIRE].

[14] M. Gualtieri, Generalized complex geometry, math/0401221 [INSPIRE].

[15] G.R. Cavalcanti and M. Gualtieri, Generalized complex geometry and T-duality, American Mathematical Society, U.S.A. (2010), arXiv:1106.1747 [INSPIRE].

[16] P. Ševera, Letters to A. Weinstein, unpublished. 
[17] P. Ševera, Poisson-Lie T-duality and Courant Algebroids, Lett. Math. Phys. 105 (2015) 1689 [arXiv: 1502.04517] [INSPIRE].

[18] T. Asakawa, H. Muraki, S. Sasa and S. Watamura, Poisson-generalized geometry and R-flux, Int. J. Mod. Phys. A 30 (2015) 1550097 [arXiv:1408.2649] [InSPIRE].

[19] R.L. Fernandes, Connections in Poisson geometry I: holonomy and invariants, J. Diff. Geom. 54 (2000) 303.

[20] T. Asakawa, H. Muraki and S. Watamura, Topological T-duality via Lie algebroids and Q-flux in Poisson-generalized geometry, Int. J. Mod. Phys. A 30 (2015) 1550182 [arXiv: 1503.05720] [INSPIRE].

[21] E. Alvarez, L. Álvarez-Gaumé and Y. Lozano, A canonical approach to duality transformations, Phys. Lett. B 336 (1994) 183 [hep-th/9406206] [INSPIRE].

[22] C. Klimčík and P. Ševera, Strings in space-time cotangent bundle and $t$ duality, Mod. Phys. Lett. A 10 (1995) 323 [hep-th/9411003] [InSPIRE].

[23] D. Roytenberg, Courant algebroids, derived brackets and even symplectic supermanifolds, math.DG/9910078.

[24] A.S. Schwarz, Geometry of Batalin-Vilkovisky quantization, Commun. Math. Phys. 155 (1993) 249 [hep-th/9205088] [INSPIRE].

[25] A.S. Schwarz, Semiclassical approximation in Batalin-Vilkovisky formalism, Commun. Math. Phys. 158 (1993) 373 [hep-th/9210115] [INSPIRE].

[26] M. Alexandrov, M. Kontsevich, A. Schwartz and O. Zaboronsky, The geometry of the master equation and topological quantum field theory, Int. J. Mod. Phys. A 12 (1997) 1405 [hep-th/9502010] [INSPIRE].

[27] N. Ikeda and X. Xu, Current algebras from DG symplectic pairs in supergeometry, arXiv: 1308.0100 [INSPIRE].

[28] N. Ikeda, Chern-Simons gauge theory coupled with BF theory, Int. J. Mod. Phys. A 18 (2003) 2689 [hep-th/0203043] [INSPIRE].

[29] C. Hofman and J.-S. Park, Topological open membranes, hep-th/0209148 [INSPIRE].

[30] D. Roytenberg, AKSZ-BV formalism and courant algebroid-induced topological field theories, Lett. Math. Phys. 79 (2007) 143 [hep-th/0608150] [InSPIRE].

[31] D. Mylonas, P. Schupp and R.J. Szabo, Membrane $\sigma$-models and quantization of non-geometric flux backgrounds, JHEP 09 (2012) 012 [arXiv: 1207.0926] [INSPIRE].

[32] A. Alekseev and T. Strobl, Current algebras and differential geometry, JHEP 03 (2005) 035 [hep-th/0410183] [INSPIRE].

[33] N. Ikeda and K. Koizumi, Current algebras and QP manifolds, Int. J. Geom. Meth. Mod. Phys. 10 (2013) 1350024 [arXiv:1108.0473] [INSPIRE].

[34] Y. Kosmann-Schwarzbach, Quasi-, twisted, and all that... in Poisson geometry and Lie algebroid theory, Progr. Math. 232 (2005) 363 [math.SG/0310359].

[35] C. Carmeli, L. Caston and R. Fioresi, Mathematical foundation of supersymmetry (with an appendix with I. Dimitrov), EMS Ser. Lect. Math., European Mathematical Society, Zürich, Switzerland (2011).

[36] V. S. Varadarajan, Supersymmetry for mathematicians: an introduction, Courant Lecture Notes Series, American Mathematical Society, U.S.A. (2004).

[37] I. Vaisman, Lectures on the geometry of Poisson manifolds, Birkhäuser, Germany (1994). 
[38] A. Deser and J. Stasheff, Even symplectic supermanifolds and double field theory, Commun. Math. Phys. 339 (2015) 1003 [arXiv:1406.3601] [INSPIRE].

[39] D. Roytenberg, A note on quasi Lie bialgebroids and twisted Poisson manifolds, Lett. Math. Phys. 61 (2002) 123 [math/0112152] [INSPIRE].

[40] D. Roytenberg, On the structure of graded symplectic supermanifolds and Courant algebroids, in Quantization, poisson brackets and beyond, T. Voronov ed., Contemporary Mathematics volume 315, American Mathematical Society, U.S.A. (2002), math/0203110 [INSPIRE].

[41] J.-S. Park, Topological open p-branes, in Symplectic geometry and mirror symmetry, K. Fukaya et al eds., World Scientific, Singapore (2001), hep-th/0012141 [INSPIRE].

[42] N. Ikeda, Deformation of BF theories, topological open membrane and a generalization of the star deformation, JHEP 07 (2001) 037 [hep-th/0105286] [INSPIRE].

[43] A.S. Cattaneo and G. Felder, On the AKSZ formulation of the Poisson $\sigma$-model, Lett. Math. Phys. 56 (2001) 163 [math/0102108] [InSPIRE].

[44] N. Ikeda and X. Xu, Canonical functions, differential graded symplectic pairs in supergeometry and Alexandrov-Kontsevich-Schwartz-Zaboronsky $\sigma$-models with boundaries, $J$. Math. Phys. 55 (2014) 113505 [arXiv: 1301.4805] [INSPIRE].

[45] N. Ikeda and K.I. Izawa, General form of dilaton gravity and nonlinear gauge theory, Prog. Theor. Phys. 90 (1993) 237 [hep-th/9304012] [INSPIRE].

[46] N. Ikeda, Two-dimensional gravity and nonlinear gauge theory, Annals Phys. 235 (1994) 435 [hep-th/9312059] [INSPIRE].

[47] P. Schaller and T. Strobl, Poisson structure induced (topological) field theories, Mod. Phys. Lett. A 9 (1994) 3129 [hep-th/9405110] [InSPIRE].

[48] C. Klimčík and T. Strobl, WZW - Poisson manifolds, J. Geom. Phys. 43 (2002) 341 [math/0104189] [INSPIRE].

[49] Y. Terashima, On Poisson functions, J. Sympl. Geom. 6 (2008) 1.

[50] M. Hansen and T. Strobl, First class constrained systems and twisting of courant algebroids by a closed 4-form, in Fundamental interactions, World Scientific (2010), arXiv:0904.0711 [INSPIRE].

[51] G. Bonelli and M. Zabzine, From current algebras for p-branes to topological M-theory, JHEP 09 (2005) 015 [hep-th/0507051] [INSPIRE].

[52] J. Grabowski and P. Urbański, Tangent lifts of Poisson and related structures, J. Phys. A 28 (1995) 6743 [math/0701076].

[53] P. Ševera and A. Weinstein, Poisson geometry with a 3 form background, Prog. Theor. Phys. Suppl. 144 (2001) 145 [math/0107133] [INSPIRE].

[54] G. Aldazabal, W. Baron, D. Marques and C. Núñez, The effective action of Double Field Theory, JHEP 11 (2011) 052 [Erratum ibid. 11 (2011) 109] [arXiv:1109.0290] [INSPIRE].

[55] D. Andriot, O. Hohm, M. Larfors, D. Lüst and P. Patalong, Non-geometric fluxes in supergravity and double field theory, Fortsch. Phys. 60 (2012) 1150 [arXiv:1204.1979] [INSPIRE].

[56] R. Blumenhagen and E. Plauschinn, Nonassociative gravity in string theory?, J. Phys. A 44 (2011) 015401 [arXiv: 1010.1263] [InSPIRE].

[57] R. Blumenhagen, A. Deser, D. Lüst, E. Plauschinn and F. Rennecke, Non-geometric fluxes, asymmetric strings and nonassociative geometry, J. Phys. A 44 (2011) 385401 [arXiv:1106.0316] [INSPIRE]. 